\title{
Tunneling Atomic Force Microscopy of Self-Assembled In(Ga)As/GaAs Quantum Dots and Rings and of GeSi/Si(001) Nanoislands
}

\author{
Dmitry Filatov1, Vladimir Shengurov', Niyaz Nurgazizov², \\ Pavel Borodin ${ }^{2}$ and Anastas Bukharaev ${ }^{2}$ \\ ${ }^{1}$ Technical Physics Research Institute, N.I. Lobachevskii University of Nizhny Novgorod, \\ 'E.K. Zavoisky Kazan' Physical-Technical Institute, Kazan' Scientific Centre, \\ Russian Academy of Sciences \\ Russia
}

\section{Introduction}

Scanning Tunnelling Microscopy (STM) has been being used for the investigation of the morphology and of the atomic structure of the semiconductor nanostructures extensively since early 1990-s (Medeiros-Ribeiro et al., 1998). More recently, STM in Ultra High Vacuum (UHV) has been applied also to the investigation of the spatial and energy distributions of the local density of states (LDOS) in the quantum semiconductor heterostructures. For example, CrossSectional STM (X-STM) has been applied to the visualization of the envelope wavefunctions of the quantum confined states in the GaSb/InAs(001) quantum wells (QWs) (Suzuki et al., 2007) and in the self-assembled InAs/GaAs(001) quantum dots (ODs) (Grandidier et al., 2000). Also, the surface InAs/GaAs(001) QDs grown by Molecular Beam Epitaxy (MBE) have been investigated by UHV STM in situ (Maltezopoulos et al., 2003). The peaks related to the quantum confined states in the QDs have been observed in the differential conductivity $\sigma_{\mathrm{d}}=$ $d I_{\mathrm{t}} / d V_{\mathrm{g}}$ spectra of the STM tip contact to the QDs (here $I_{t}$ is the tip current and $V_{g}$ is the gap voltage). The $\sigma_{\mathrm{d}}(x, y)$ images of the ODs ( $x$ and $y$ are the tip coordinates on the sample surface) recorded at the values of $V_{\mathrm{g}}$ corresponding to the peaks in the $\sigma_{\mathrm{d}}\left(V_{\mathrm{g}}\right)$ spectra correlated with the probability density patterns $|\chi(x, y)|^{2}$ where $\chi(x, y)$ are the lateral components of the electron quantum confined states envelope wavefunctions calculated for the pyramidal InAs/GaAs(001) ODs defined by the (101) facets (Stier et al., 1999).

The present chapter is devoted to the investigation of the electronic states in the selfassembled semiconductor nanostructures [namely, the InAs/GaAs(001) QDs, the InGaAs/GaAs(001) quantum rings (QRs), and the GeSi/Si(001) nanoislands] by Tunnelling Atomic Force Microscopy (AFM). The samples with the surface self-assembled semiconductor nanostructures were scanned across by a conductive Si AFM probe covered by a conductive coating $\left(\mathrm{Pt}, \mathrm{W}_{2} \mathrm{C}\right.$, or a diamond-like film) in the contact mode. The bias voltage $V_{\mathrm{g}}$ was applied between the AFM probe and the sample. Simultaneously with the acquisition of the topography $z(x, y)$, the $I-V$ curves of the probe-to-sample contact $I_{\mathrm{t}}\left(V_{\mathrm{g}}\right)$ were acquired in each point in the scans. 
Earlier, Tunnelling AFM has been applied mainly to the characterization of the local electrical properties of the thin dielectric films on the conductive substrates (Yanev et al., 2008). Also, Tunnelling AFM in UHV has been applied to the tunnel spectroscopy of individual $\mathrm{Au}$ nanoclusters in the ultrathin $\mathrm{SiO}_{2} / \mathrm{Si}$ films (Zenkevich et al., 2011). The present chapter summarizes a series of the original studies where the authors have applied Tunnelling AFM to the mapping of the LDOS in the self assembled semiconductor nanostructures for the first time (Filatov et al., 2010, 2011, Borodin et al., 2011). The main advantage of Tunnelling AFM compared to UHV STM is that the former allows the ex situ investigation of the surface semiconductor nanostructures covered by a native oxide layer during the sample transfer from the growth setup to the AFM one through the ambient air. This makes the STM studies of these samples hardly possible.

Another distinctive feature of the studies present in this chapter is that the samples with InGaAs/GaAs(001) QDs and QRs have been grown by Atmospheric Pressure Metal Organic Vapour Phase Epitaxy (AP-MOVPE). In most studies reported in the literature, the QDs grown by MBE or by Low Pressure (LP) MOVPE have been investigated. Nevertheless, the investigations of the electronic properties of the InAs/GaAs(001) QDs grown by AP MOVPE are of a considerable interest because this growth method is more promising for the commercial device production due to its lower cost and higher productivity as compared to MBE and LP-MOVPE.

Also, the structures with the self-assembled GeSi/Si(001) nanoislands studied in the present chapter were grown by a novel technique of Sublimation MBE (SMBE) in $\mathrm{GeH}_{4}$ ambient. In this method, the Si layers are grown from an ordinary sublimation source in UHV. To deposit $\mathrm{Ge}, \mathrm{GeH}_{4}$ is introduced in the growth chamber at the pressure of $\sim 10^{-2} \div 10^{-4}$ Torr and undergo pyrolysis on the heated substrate. So far, this method is some hybrid between the conventional MBE from the sublimation source and LP VPE. Again, in the majority of works, the GeSi/Si(001) nanoislands grown by MBE have been studied (Berbezier \& Ronda, 2009). The main advantage of the hybrid technique of $\mathrm{SMBE}$ in $\mathrm{GeH}_{4}$ ambient as compared to the ordinary VPE of Si and Ge from silanes and germanes, respectively is that SMBE allows growing the $\mathrm{Si}$ layers of high crystalline quality and purity at relatively low temperatures (450 $\div 500^{\circ} \mathrm{C}$ ) keeping the high enough growth rates. In addition, SMBE offers a broader choice of the doping impurities as well as a wider range of their concentrations achievable than VPE. On the other hand, the deposition of Ge from a gaseous precursor provides higher uniformity of the nominal thickness of the deposited Ge layer $d_{\mathrm{Ge}}$ over the substrate surface.

Having applied Tunnel AFM to the investigation of the LDOS in the self assembled semiconductor nanostructures described above, we have observed the patterning of the probe current images $I_{t}(x, y)$ of the InAs/GaAs(001) QDs and of the InGaAs/GaAs(001) QRs as well as the peaks in the $\sigma_{\mathrm{d}}(x, y)$ spectra of the contact of the AFM probe to the sample surface attributed respectively to the spatial and energy distributions of the LDOS in the quantum heterostructures. The results of the LDOS mapping by Tunnelling AFM have been compared to the results of the calculations of the probability density patterns $|\chi(x, y)|^{2}$ reported in the literature. The Tunnel AFM data allowed the identification of the quantum confined states in the InAs/GaAs(001) QDs grown by AP MOVPE the interband optical transitions between which are manifested in the photosensitivity (PS) spectra of the QD structures. Finally, the direct measurements of the LDOS spectrum in the self assembled $\mathrm{Ge}_{x} \mathrm{Si}_{1-x} / \mathrm{Si}(001)$ nanoislands demonstrated the type I conduction band alignment in them at $x<0.45$. 


\section{Self assembled InAs/GaAs(001) quantum dots}

\subsection{Growth and characterization}

In this subsection, the details of the growing the samples for the Tunnelling AFM investigation by AP MOVPE and of their characterization are presented.

The InAs/GaAs(001) QD structures for the tunnel spectroscopy of the LDOS in the conduction band were grown on the $n^{+}-\mathrm{GaAs}(001)$ substrates by Dr. B. N. Zvonkov in Research Institute for Physics and Technology, N. I. Lobachevskii University of Nizhny Novgorod, Russia using a homemade setup for AP MOVPE from trimethylgallium, trimethylindium, and $\mathrm{AsH}_{3}$. The schematic of the QD structures for the Tunnelling AFM investigations is shown in Fig. 1, a. The substrates were misoriented from (001) by $3 \div 5^{\circ}$ towards $<110\rangle$. The donor concentration in the substrate material was $\sim 10^{18} \mathrm{~cm}^{-3}$. The $\mathrm{GaAs}$ buffer layers with the thickness $d_{\mathrm{b}} \approx 200 \mathrm{~nm}$ were doped by Si heavily up to the donor concentration $\sim 10^{18} \mathrm{~cm}^{-3}$ using pulsed laser sputtering of a bulk Si target placed in the MOVPE reactor. More detains on this growth technique can be found elsewhere (Karpovich et al., 2004a). The $3 \mathrm{~nm}$ thick intentionally undoped GaAs spacer layers were grown between the $n^{+}-\mathrm{GaAs}$ buffers and the InAs ODs. The latter were grown at the substrate temperature $T_{\mathrm{g}}=530^{\circ} \mathrm{C}$, the nominal thickness of the InAs layers $d_{\text {InAs }}$ was $\approx 5$ monolayers (ML).

The morphology of the grown samples was first examined by ambient air AFM using NT MDT® Solver Pro ${ }^{\mathrm{TM}}$ instrument in Contact Mode. The NT MDT® CSG-01 silicon AFM probes were used. The curvature radii of the probe tips $R_{\mathrm{p}}$ were $<10 \mathrm{~nm}$ (according to the vendor's specifications).

The morphology of the surface QD arrays was characterized quantitatively by the following parameters:

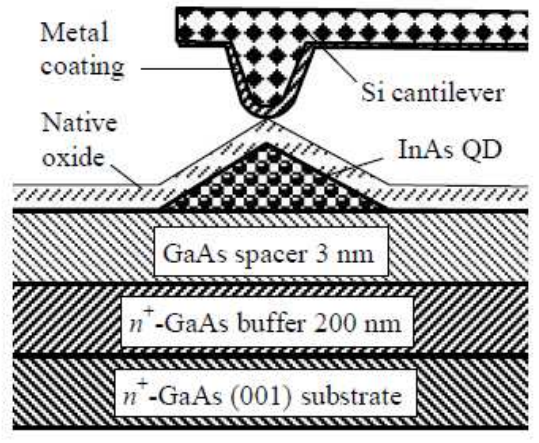

$a$

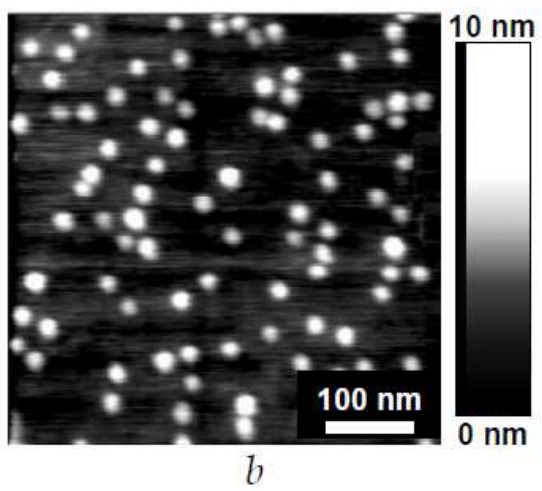

Fig. 1. The schematic ( $a$ ) and an ambient air AFM image $(b)$ of a structure with the InAs $/ n$ GaAs/ $n^{+}-\mathrm{GaAs}(001)$ surface QDs. 
- $\quad$ The average height of the QDs $<h>$

- $\quad$ The averaged diameter of the QDs $\langle D>$. The value of $D$ for given QD was defined as $D$ $=P / 2 \pi$ where $P$ was the perimeter of the AFM image of the QD measured at the level of $0.1<h>$ above the surface of the wetting layer

- $\quad$ The surface density of the QDs $N_{\mathrm{s}}$

The morphological parameters of the QD arrays listed above were determined by the digital processing of the AFM data. In order to identify the QDs on the surface of the wetting layer, a threshold particle recognition algorithm has been applied.

The key issue in the investigations of the morphology of the surface InAs/GaAs(001) QDs by AFM was the effect of convolution (Bukharaev et al., 1999) originating from a relatively large AFM probe tip radius $R_{\mathrm{p}}(\sim 10 \mathrm{~nm})$ as compared to the typical sizes of the InAs/GaAs(001) QDs defined by the (101) facets $(D=12 \div 18 \mathrm{~nm}, h=5 \div 6 \mathrm{~nm})$. In order to extract the actual size and shape of the InAs/GaAs(001) QDs grown by AP MOVPE from the AFM data, we have applied the digital processing of the AFM images using an original software for the correction of the convolution artifacts (so called "deconvolution"). This software utilizes the "virtual AFM" algorithm (Bukharaev et al., 1998).

In order to apply this algorithm to the correction of the convolution artifacts, one needs to know the exact geometry of the actual probe tip used in the experiment. The specifications provided by the AFM cantilevers' vendors appear to be insufficient often. The actual probe tip shape may be determined by the measuring of a standard sample with the geometry known a priori. However, the theory of the convolution artifacts sets up an essential requirement on the geometrical parameters of the standard sample to be close to these ones of the samples under study [i. e. the InAs/GaAs(001) QDs in our case]. In order to evaluate the AFM tip shape, we have used a structure with the surface self assembled GeSi/Si(001) pyramidal-shaped nanoislands (Medeiros-Ribeiro et al., 1998) as a standard sample. The structure was grown by Dr. A. V. Novikov, Institute for Physics of Microstructures, Russian Academy of Sciences (Nizhny Novgorod, Russia) using standard MBE. The self assembled GeSi/Si(001) pyramidal-shaped nanoislands are defined by the (105) facets, their heigt can be extracted just from the AFM data directly. Note that the convolution artifacts do not affect the acuracy of the measurements of the heights $h$ of the GeSi/Si(001) nanoislands as well as of the InAs/GaAs(001) QDs. So far, all the parameters needed to determine the actual probe shape can be determined from a single AFM scan of a single GeSi/Si(001) pyramidal island.

Another ulitmate requrement is that the hieght of the topographic elements on the standard sample should exceed the heihgts of the invesitgated objects [namely, the InAs/GaAs(001) QDs]. The self assembled GeSi/Si(001) pyramid islands satisfy this requirement as thier height $h$ may reach $\sim 10 \mathrm{~nm}$ when grown in the appropriate conditions, that is well enough for the InAs/GaAs(001) QDs (typically, $h=5 \div 6 \mathrm{~nm}$ ). So far, the GeSi/Si(001) pyramid nanaoislands appear to be a good natural standard for the measurement of the probe shape in the particular case of the deconvolution of the AFM images of the self-assembled InAs/GaAs(001) QDs.

The raw images of the QDs seemd round, their lateral sizes $D$ were 30 to $50 \mathrm{~nm}$ (Fig. 2, a). In the AFM images after the deconvolution (Fig. 2, b) the (101) faceting of the InAs/GaAs(001) QDs grown by AP MOVPE is seen clearly. 

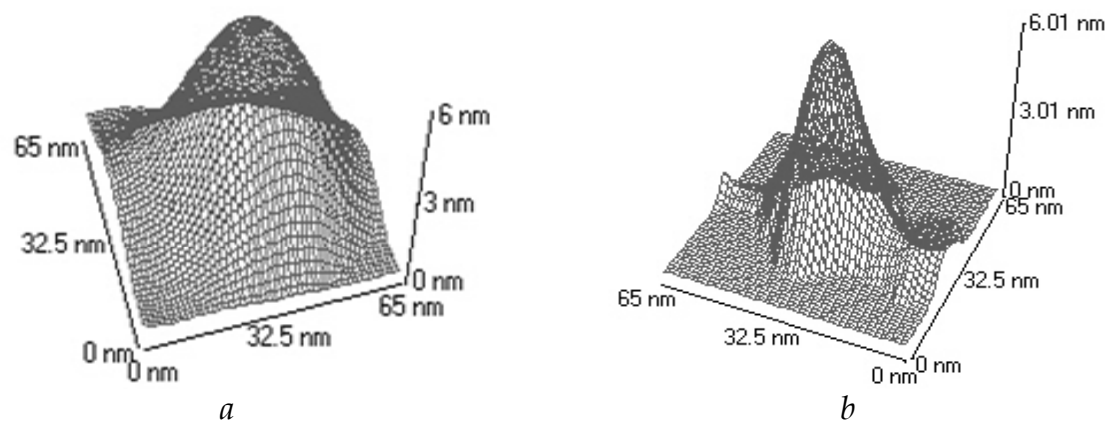

Fig. 2. The AFM images of a surface InAs/GaAs(001) QD grown by AP MOVPE: as measured $(a)$ and after the deconvolution $(b)$.

The base sides were directed along $<110>$, their length $b=14 \div 18 \mathrm{~nm}$ like the ones grown by MBE (Ledentsov et al., 1999, Maltezopoulos et al., 2003). The top of the pyramid was truncated slightly (Fig. 2,b) that could be explained noting that the top of the pyramid is a concentrator of the tensile strain. So far, the truncation of the pyramid reduces the overall elastic energy of the QD. Similar shape of the QD tops has been observed by UHV STM (Maltezopoulos et al., 2003). Therefore, the aspect ratio $A_{\mathrm{R}}$ of the InAs/GaAs(001) QDs is less than 1:2 slightly.

The size quantization energy spectrum of the InAs/GaAs(001) QDs grown by AP MOVPE has been examined by photoluminescence (PL) spectroscopy at $77 \mathrm{~K}$ and by the photoelectric spectroscopy at 300K. The structures for the PL spectroscopy have been grown on the semiinsulating $\mathrm{GaAs}(001)$ substrates, the buffer layers have been undoped intentionally. The InAs QDs in these structures have been capped by a $30 \mathrm{~nm}$ thick GaAs cladding layer. The structures for the photoelectric measurements had the same design except the substrates were form $n^{+}$-GaAs. The InAs in all three types of the structures (for Tunnelling AFM, for $\mathrm{PL}$, and for the photoelectric spectroscopy) have been grown in the same conditions $\left(T_{\mathrm{g}}=\right.$ $530^{\circ} \mathrm{C}, d_{\text {InAs }}=5 \mathrm{ML}$ ). The details of the measurement techniques as well as the analysis of the experimental results can be found elsewhere (Karpovich et al., 2004b).

\subsection{The conduction band states}

In this subsection, the results of the Tunnelling AFM investigations of the quantum confined electron states in the surface InAs QDs are presented and discussed. Also, the results of the studies of the laterally coupled surface InAs QDs are presented.

The Tunnelling AFM studies were carried out at 300K using Omicron ${ }^{\circledR}$ MultiProbe $\mathrm{P}^{\mathrm{TM}}$ UHV system equipped by Omicron ${ }^{\circledR}$ UHV VT AFM/STM. A typical topographic image $z(x$, $y$ ) and the probe current one $I_{\mathrm{t}}(x, y)$ of an InAs/GaAs(001) QD sample are presented in Fig. $3, a \& b$, respectively. An increasing of $I_{\mathrm{t}}$ every time the AFM tip encounters the QD surface had been observed. This observation has been attributed to the electron tunnelling between the AFM tip and the conductive buffer layer through the quantum confined states in the QDs (a qualitative band diagram of a contact of a Pt coated AFM tip to an InAs/GaAs $/ n^{+}-$ GaAs biased negatively is presented in Fig. $4, b$ ). 

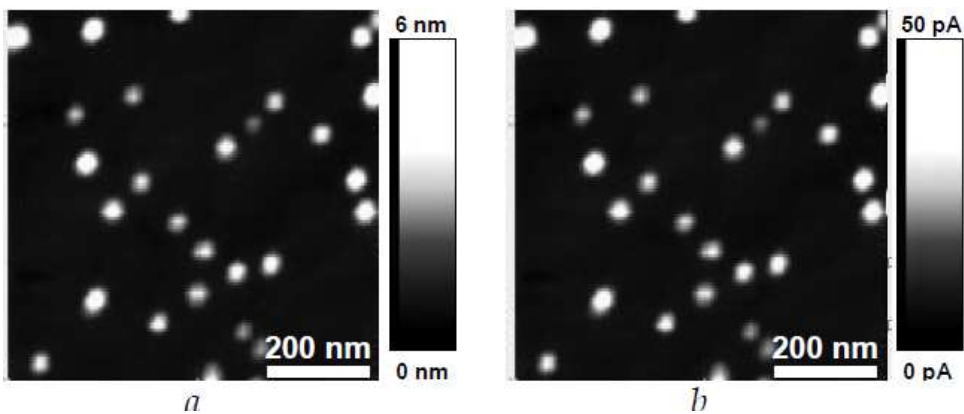

Fig. 3. The topographic $(a)$ and the probe current $(b)$ images of the surface InAs/GaAs $/ n^{+}-$ GaAs(001) QDs. $V_{\mathrm{g}}=-3.7$ V. Reproduced from (Filatov et al., 2010) under license by IoP Publishing Ltd.

The differential conductivity spectra $\sigma_{\mathrm{d}}\left(V_{\mathrm{g}}\right)=d I_{\mathrm{t}} / d V_{\mathrm{g}}$ of the tunnel contact of a Pt coated AFM tip to different points of the surface an InAs/GaAs $/ n^{+}-\mathrm{GaAs}(001)$ QD are presented in Fig. 4. , $a$. The $\sigma_{\mathrm{d}}\left(V_{\mathrm{g}}\right)$ spectra have been calculated from the measured $I_{\mathrm{t}}\left(V_{\mathrm{g}}\right)$ curves of the probe-to-sample contact by the numerical differentiation with the nonlinear smoothing. The points on the QD surface where the respective $I_{\mathrm{t}}\left(V_{\mathrm{g}}\right)$ curves had been measured are marked in Fig. 5, $a$. The peaks observed in the $\sigma_{\mathrm{d}}\left(V_{\mathrm{g}}\right)$ spectra were attributed to the tunnelling of the electrons between the metallic tip coating and the conductive substrate through the quantum confined states in the QDs (Fig. $4 ., b$ ). The native oxide on the sample surface formed a potential barrier, the second triangle potential barrier was formed by the depletion layer of the contract of the metal tip coating to the GaAs $/ n^{+}-$GaAs. In the UHV STM studies (Maltezopoulos et al., 2003) the first potential barrier was formed by the vacuum gap between the metal STM probe and the sample surface.
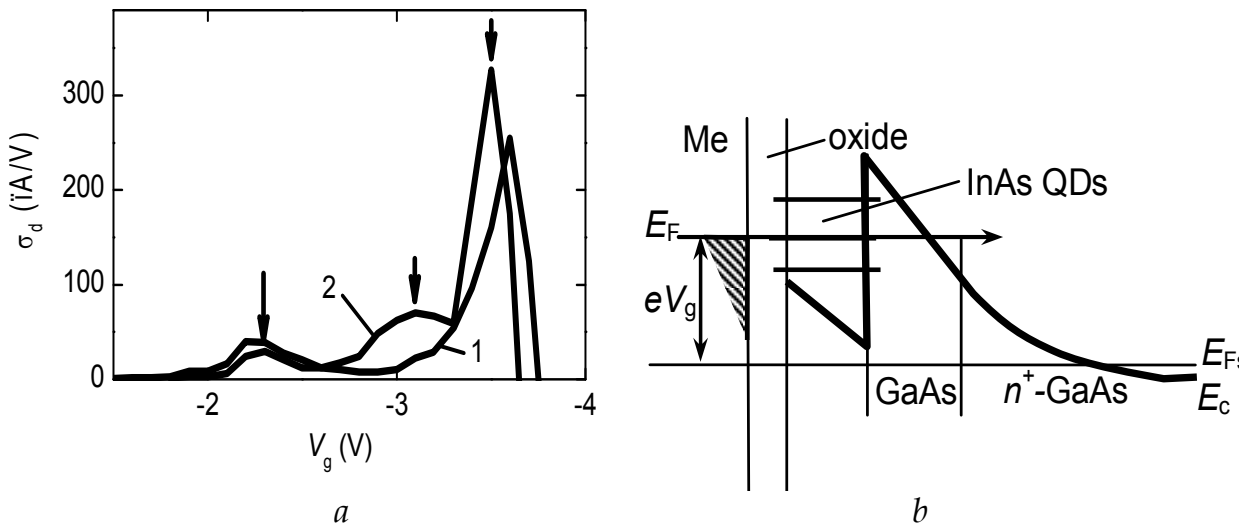

Fig. 4. The differential conductivity spectra $\sigma_{\mathrm{d}}\left(V_{\mathrm{g}}\right)(a)$ and the qualitative band diagram $(b)$ of a negatively biased contact of a metal coated AFM tip to an InAs QD on the $n$-GaAs $/ n^{+}-$ GaAs (001) substrate. The curve numbers denote the points of the $I-V$ curves' measurements marked in Fig. 5, a. Reproduced from (Filatov et al., 2010) with permission from $\odot$ Pleiades Publishing, Ltd. 


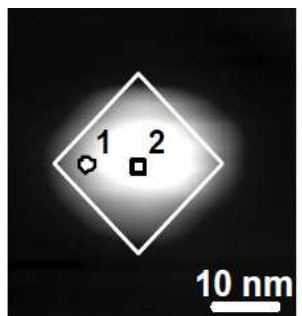

a

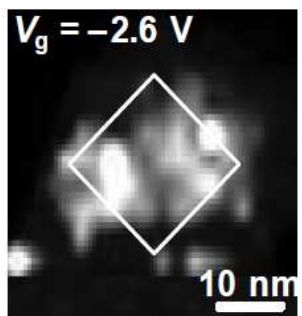

b

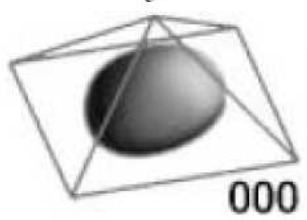

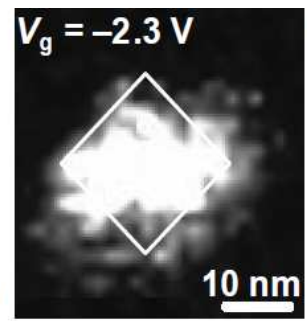

C

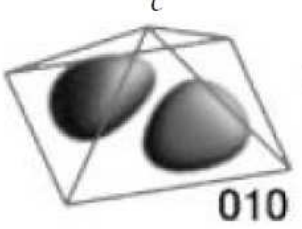

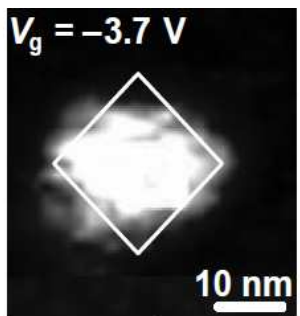

d

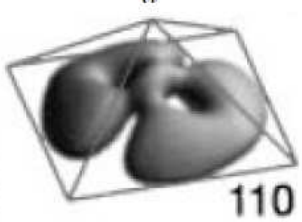

Fig. 5. The UHV AFM $(a)$ and the probe current $(b-d)$ images of an InAs QD on the $n$ $\mathrm{GaAs} / n^{+}-\mathrm{GaAs}(001)$ substrate. Reproduced from (Filatov et al., 2010) with permission from (CPleiades Publishing, Ltd. Below, the surfaces of the equal probability density $|\chi(x, y, z)|^{2}=$ 0.65 calculated for several quantum confined electron states $\mid n_{1} n_{2} n_{3}>$ in a pyramidal InAs/GaAs(001) QD with the base side length $b=16 \mathrm{~nm}$ are presented. Reproduced partly from (Stier et al., 1999) with permission from (CAmerican Physical Society.

The AFM and the probe current images of an individual InAs $/ n^{+}-\mathrm{GaAs}$ QD are presented in Fig. 5. Note that because of the convolution effect, the AFM image of the QD is rounded and enlarged as compared to the actual QD size revealed using the deconvolution $(b=14 \div 16$ $\mathrm{nm}$, see Sec. 2.1 above).

Following (Maltezopoulos et al., 2003), the patterns of the $I_{t}(x, y)$ images at certain values of $V_{\mathrm{g}}$ corresponding to the maxima of the $\sigma_{\mathrm{d}}\left(V_{\mathrm{g}}\right)$ spectra in Fig. 4, $a$ have been related to the spatial distribution of the LDOS in the $(x, y)$ plane:

$$
\rho_{\mathrm{E}}(\mathrm{x}, \mathrm{y}) \propto \sum_{\mathrm{n}_{1} \mathrm{n}_{2} \mathrm{n}_{3}=0}^{\mathrm{N}_{1} \mathrm{~N}_{2} \mathrm{~N}_{3}}\left|\chi_{\mathrm{n}_{1} \mathrm{n}_{2} \mathrm{n}_{3}}(\mathrm{x}, \mathrm{y})\right|^{2} .
$$

Here the envelope wavefunctions $\chi(x, y)$ were considered to be spin-independent and twofold spin-degenerated. The summation in (1) was taken over the states below the Fermi level in the probe coating material $E_{\mathrm{F}}$. In other words, in the case of the QDs grown on the n$\mathrm{GaAs} / n^{+}-\mathrm{GaAs}(001)$ substrate the energies of the respective quantum confined states must satisfy the following condition:

$$
\mathrm{E}_{\mathrm{n}_{1} \mathrm{n}_{2} \mathrm{n}_{3}}<\mathrm{E}_{\mathrm{Fs}}+\mathrm{eV}_{\mathrm{g}},
$$

where $E_{\mathrm{Fs}}$ is the Fermi level in the $n^{+}$-GaAs buffer ( see Fig. $4 ., b$ ). Condition (2) defines the upper limits of the summation $N_{1}, N_{2}$, and $N_{3}$ in (1).

Again, following (Maltezopoulos et al., 2003), the tunnel current images of the QDs $\mathrm{I}_{\mathrm{t}}(x, y)$ were compared to the probability density patterns $|\chi(x, y, z)|^{2}=$ const calculated for the quantum confined electron states in the pyramidal InAs/GaAs(001) QDs (Stier et al., 1999). 
The probe current images of the QDs obtained by Tunnelling AFM were more noisy than the ones obtained by STM in UHV (Maltezopoulos et al., 2003) that was attributed to the nonuniformity of the thickness of the native oxide covering the QDs. Nevertheless, several electron quantum confined states in the QDs were identified, the respective images of the $|\chi(x, y, z)| 2=0.65$ surfaces (Stier et al., 1999) are shown in Fig. 5.

In order to associate the spectral positions of the peaks in the $\sigma_{\mathrm{d}}\left(V_{\mathrm{g}}\right)$ spectra with the quantum confined level energies $E_{n_{1} n_{2} n_{3}}$, one must take into account the partial voltage drop on the depletion layer between the QDs and the $n^{+}$-GaAs buffer layer as well as the one on the surface states at the semiconductor/native oxide interface. Following (Suzuki et al., 2007), we have applied a simple one-dimensional model based on the solution of onedimensional Poisson's equation (Feenstra \& Stroscio, 1987) to account for the voltage drop on the depletion layer of the probe-to-sample contact. In order to account for the surface charge density on the surface states on the interface between the sample surface and the native oxide, we have applied Hasegawa's model (Hasegawa \& Sawada, 1983). The calculations have shown that approximately $1 / 2$ of $V_{\mathrm{g}}$ drops on the surface states.

The InAs/GaAs (001) surface QD structures grown by AP MOVPE are featured by the presence of a considerable number of the QDs arranged in the pairs along the growth steps in a close proximity to each other (see Fig. 1, $b$ ) that may result in a considerable overlap of the envelope wavefunctions of the quantum confined states in the adjacent QDs. An example of the UHV AFM image as well as a series of the probe current images of a pair of the laterally coupled surface InAs/GaAs(001) QDs are presented in Fig. 6.
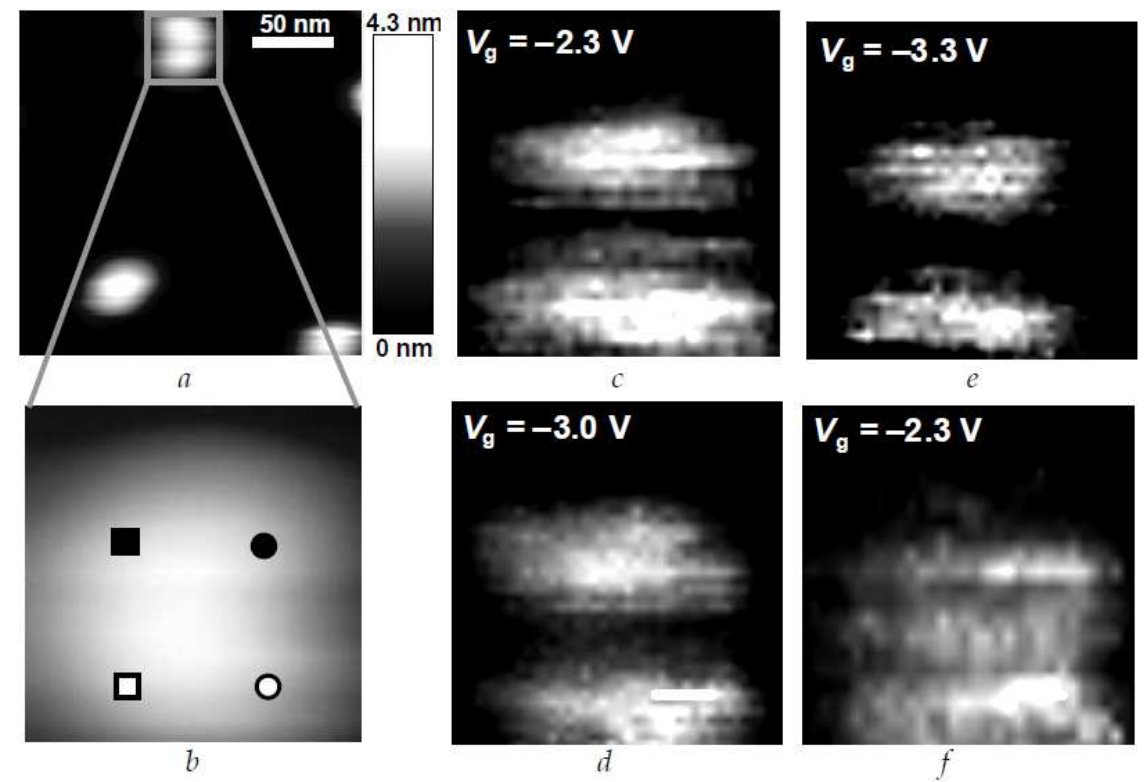

Fig. 6. The UHV AFM $(a, b)$ and the probe current $(c-f)$ images of the laterally coupled surface InAs/GaAs(001) QDs. The symbols in fig. (b) mark the points of the measurement of the respective tunnel spectra presented in Fig. 7. 


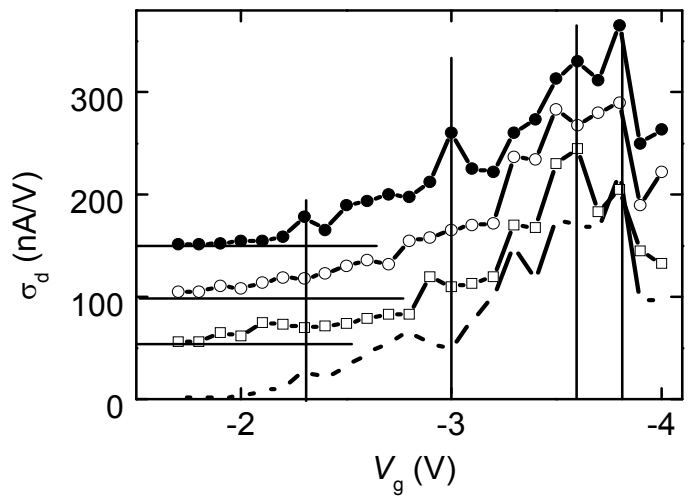

Fig. 7. The differential conductivity spectra $\sigma_{\mathrm{d}}\left(V_{\mathrm{g}}\right)$ of the tunnel contact of a Pt coated AFM tip to the laterally coupled InAs $/ n-\mathrm{GaAs} / n^{+}-\mathrm{GaAs}(001)$ QDs. The points of measurement of the initial $I-V$ curves are denoted in Fig. $6, b$ by the respective symbols.

The differential conductivity spectra $\sigma_{\mathrm{d}}\left(V_{\mathrm{g}}\right)$ of the tunnel contact of a Pt coated AFM tip to the laterally coupled InAs $/ n-\mathrm{GaAs} / n^{+}-\mathrm{GaAs}(001)$ QDs are presented in Fig. 7. The asymmetry of the current images in Fig. 6, $c-f$ (as compared to the ones of the single QDs, see Fig. 5, $b-d)$, which had varied with increasing $V_{\mathrm{g}}$ along with the splitting of the peaks related to the quantum confined states in the coupled QDs (Fig. 7) were attributed to the hybridization of the quantum confined states in the laterally coupled QDs. Similar patterns of the tunnel current images and tunnel spectra as well as of their dynamics with varying $V_{\mathrm{g}}$ have been observed while studying the hybridization of the quantum confined states in the $\mathrm{GaSb} / \mathrm{InAs}(001)$ double symmetric QWs by X-STM in UHV (Suzuki et al., 2007).

\subsection{The valence band states}

In this subsection, the results of the Tunnelling AFM studies of the hole quantum confined states in the InAs/GaAs(001) QDs are presented. It is worth noting that the authors of the present chapter had applied Scanning Probe Microscopy technique for the studying of the valence band states in the InAs/GaAs(001) self assembled QDs for the first time.

The InAs/GaAs(001) QD samples for the investigation of the valence band states in the InAs QDs by Tunneling AFM have been grown on the $p^{+}-\mathrm{GaAs}(001)$ substrates. The GaAs buffer layers were also doped heavily by Zn from diethylzinc up to the acceptor concentration $N_{A}$ $\sim 10^{18} \mathrm{~cm}^{-3}$. The intentionally undoped $3 \mathrm{~nm}$ thick GaAs spacer layers were grown prior to the deposition of InAs, as in the InAs/GaAs $/ n^{+}-\mathrm{GaAs}(001)$ QD structures for the investigations of the electron states described in the previous subsection. The technological parameters of the process of growing the InAs QDs were the same, as in the case of the structures grown on the $n^{+}-\mathrm{GaAs}$ substrates: $T_{\mathrm{g}}=530^{\circ} \mathrm{C}, d_{\mathrm{InAs}}=5 \mathrm{ML}$.

The differential conductivity spectra $\sigma_{\mathrm{d}}\left(V_{\mathrm{g}}\right)$ of the tunnel contact of a Pt coated AFM tip to an InAs QD on the GaAs/ $p^{+}$-GaAs (001) substrate are presented in Fig. 8, $a$. The oscillations of the $\sigma_{\mathrm{d}}\left(V_{\mathrm{g}}\right)$ spectra have been attributed to the tunneling from the valence band states in the $p^{+}-\mathrm{GaAs}$ buffer to the free states above the Fermi level in the metal tip coating through the quantum confined hole states in the InAs QD (Fig 8, $b$ ). 

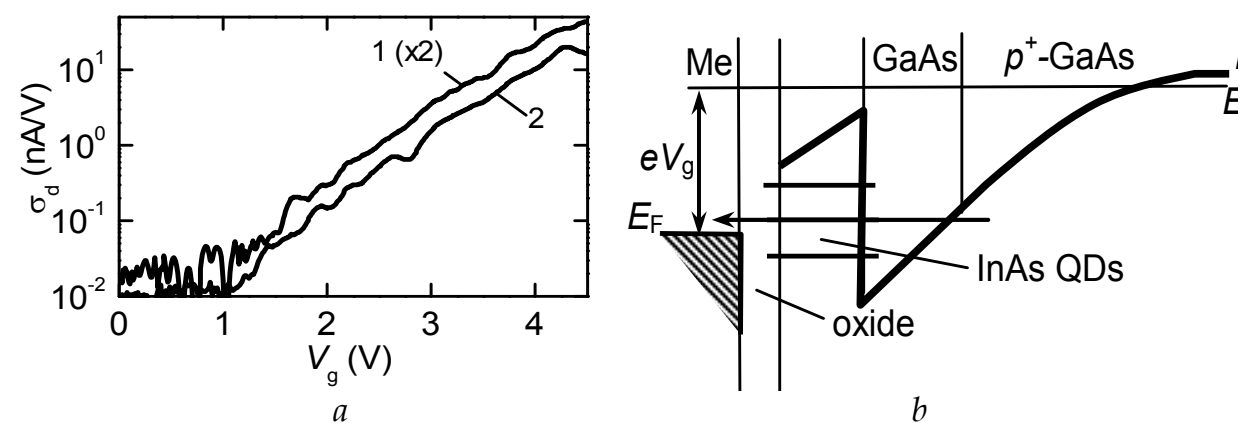

Fig. 8. The differential conductivity spectra $\sigma_{\mathrm{d}}\left(V_{\mathrm{g}}\right)(a)$ and the qualitative band diagram $(b)$ of the positively biased contact of a Pt coated AFM tip to an InAs QD on the GaAs $/ p^{+}-\mathrm{GaAs}$ (001) substrate. The curve numbers denote the points of the initial $I-V$ curves' measurements shown in Fig 9, a. Reproduced partly from (Filatov et al., 2010) under license from $@$ IoP Publishing, Ltd.

Comparing the $\sigma_{\mathrm{d}}\left(V_{\mathrm{g}}\right)$ spectra of the QDs on the $p^{+}$-GaAs (Fig. 8, $\left.a\right)$ with the ones of the QDs grown on $n^{+}$-GaAs (Fig $4, a$ ), one can note that the peaks related to the tunnelling via the quantum confined electron states in the QDs were well resolved in the case of the InAs/ $n^{+-}$ GaAs QDs while a nearly exponential curves with the weak oscillations only have been observed in the InAs $/ p^{+}$-GaAs QDs(Fig. 8, a). This observation could be explained noting that according to (Stier et al., 1999), the energy spacing between the electron levels in the InAs/GaAs(001) QDs with the base size length $b=15 \div 20 \mathrm{~nm}$ is $\sim 100 \mathrm{meV}$ while the one for the hole levels is $10 \div 20 \mathrm{meV}$ only. As a result, the peaks in the $\sigma_{\mathrm{d}}\left(V_{\mathrm{g}}\right)$ spectra related to the quatum confined hole states in the InAs QDs were resolved poorly because of the thermal and structural broadening. Note also that the spectral spacing between the peaks in the $\sigma_{\mathrm{d}}\left(V_{\mathrm{g}}\right)$ spectra ascribed to the quantum confined hole states in Fig. 8, $a$ was much less than the one for the conduction band states (Fig. 4, a), that agrees with proposed interpretation of the QDs' tunnel spectra as well.

The AFM and the probe current images of an individual InAs QD on the GaAs $/ p^{+}-$ $\mathrm{GaAs}(001)$ substrate are presented in Fig. 9. The probe current images $I_{t}(x, y)$ of the QD measured at different values of $V_{\mathrm{g}}$ were related to the lateral spatial distribution of the LDOS of the quantum confined hole states in the QD $\rho_{\mathrm{E}}(x, y)$ at the respective vaules of $E$. In the case of the InAs QDs grown on the GaAs/ $p^{+}-\mathrm{GaAs}(001)$ substrate, the hole quantum confined states, the energy of which satisfies the following condition:

$$
\mathrm{E}_{\mathrm{Fs}}-\mathrm{eV}_{\mathrm{g}}<\mathrm{E}_{\mathrm{n}_{1} \mathrm{n}_{2} \mathrm{n}_{3}}<\mathrm{E}_{\mathrm{Fs}}
$$

where $E_{\mathrm{Fs}}$ is the Fermi level energy in the $p^{+}$-GaAs buffer (see the band sketch in Fig. 8, $a$ ) can manifest themselves in the probe current images $I_{t}(x, y)$.

Again, the maps of the probe current $I_{\mathrm{t}}(x, y)$ have been compared to the equal probability density patterns $|\chi(x, y, z)|^{2}=$ const calculated for several lower quantum confined hole states (Stier et al., 1999). Although the quality of the probe current images was not so good, several quantum confined states have been identified (Fig. 9). 


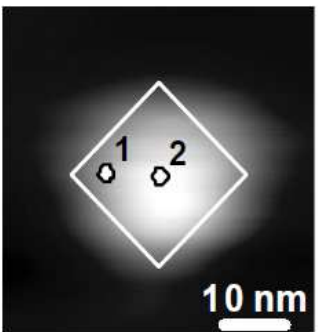

$a$

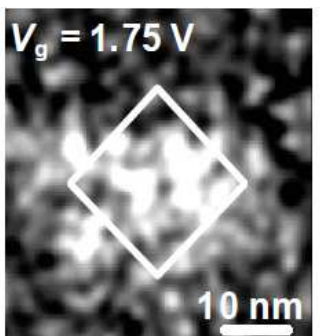

b

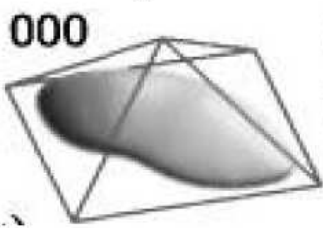

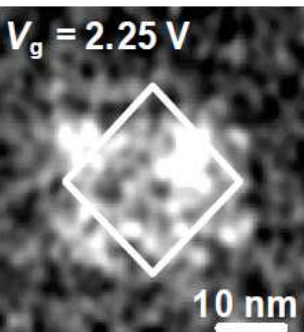

C

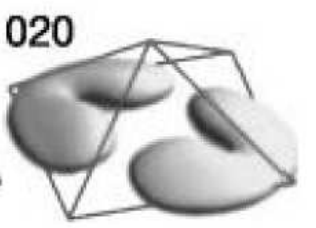

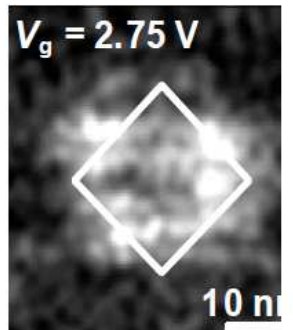

d

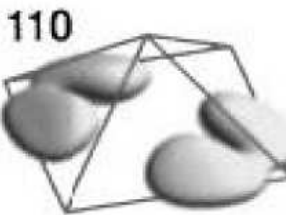

Fig. 9. The AFM $(a)$ and the probe current $(b-d)$ images of an InAs QD on the GaAs $/ p^{+}-$ GaAs(001) substrate. Reproduced partly from (Filatov et al., 2010) under license from @IoP Publishing, Ltd. Below the surfaces of equal probability density $|\chi(x, y, z)|^{2}=0.65$ calculated for several quantum confined hole states $\mid n_{1} n_{2} n_{3}>$ in a pyramidal InAs/GaAs(001) QD with the base side length $b=16 \mathrm{~nm}$ are presented. Reproduced partly from (Stier et al., 1999) with permission from (CAmerican Physical Society.

Having identified the quantum confined electron and hole states in the InAs/GaAs(001) QDs grown by AP MOVPE, we succeeded to identify the states the interband optical transition between which are manifested in the PS spectra of the QD structures grown by AP MOVPE.

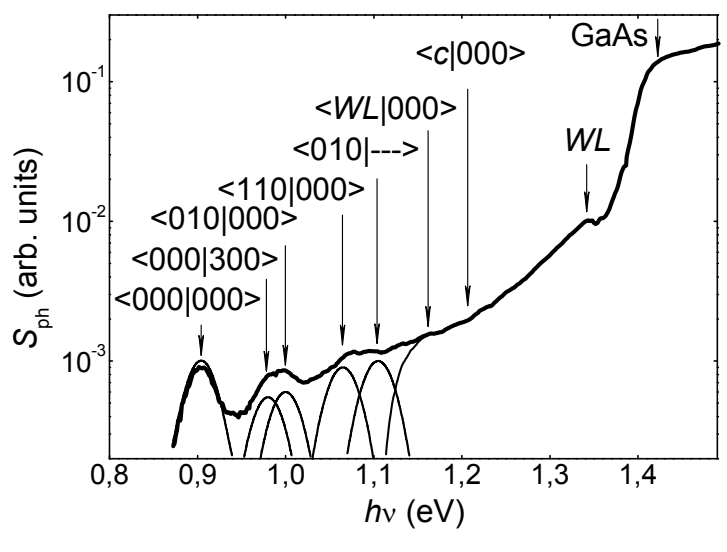

$a$

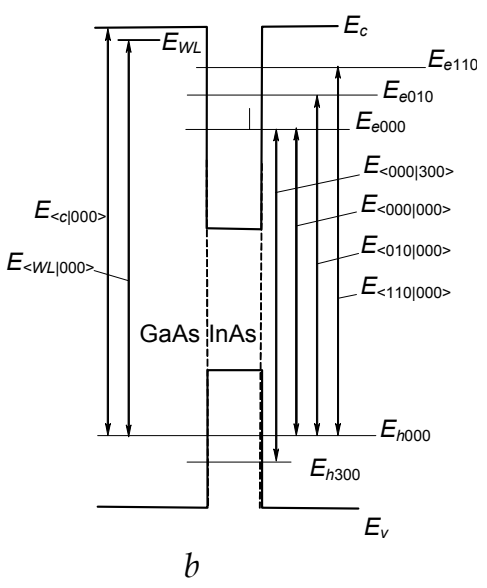

$b$

Fig. 10. The photosensitivity spectrum (a) and the band diagram (b) (300K) of an InAs/GaAs(001) QD structure grown by AP MOVPE. Reproduced from (Filatov et al., 2010) with permission from $\bigcirc$ Pleiades Publishing Ltd. 
An example of the PS spectrum $S_{\mathrm{ph}}(h v)$ of an InAs/GaAs(001) QD structure measured by the photovoltage spectroscopy in a liquid electrolyte (Karpovich et al., 2004b) is presented in Fig. $10, a$. The QDs in this particular structure were grown in the same conditions as in the samples for the Tunnelling AFM investigstions described above. Several peaks related to the interband optical transitions between the quantum confined electron and hole states in the QDs are present in the PS spectrum. Also, the PS bands related to the transitions from the ground hole state in the QD $\mid 000>$ to the ground electron states in the InAs wetting layer $\mid W L>$ and to the conduction band states in GaAs have been observed. The band diagram of an InAs/GaAs QD structure with the transitions manifested in the PS spectrum (Fig. 10, a) shown schematically is presented in Fig. 10, $b$. This diagram was based on the best fit between the interband transition energies extracted from the PS spectrum in Fig. 10, $a$ and the ones calculated from the data on the quantum confined level energies in the pyramidal InAs/GaAs(001) QDs as the function of the QD base size $b$ (Stier et al., 1999). The best fit was found at $b \approx 16 \mathrm{~nm}$ that is consistent with the ambient air AFM data obtained using the deconvolution (see Sec. 2.1). Note that many possible transitions are not manifested in the PS spectrum, because the overlap integrals between the envelopes of the respective electron and hole states are close to zero (Stier et al., 1999). Only the transitions between the states the overlap integrals for which are close to unity are manifested in the PS spectrum.

We have failed to identify one of the higher energy hole states marked as |---> in Fig. 10 because the data for the respective hole energy band are not presented in the publication by (Stier et al,. 1999). In the other aspects, the data on the morphology, on the electronic structure, and on the energy spectrum of the InAs/GaAs(001)QDs grown by AP MOVPE provided by ambient air and Tunnelling AFM, by the PL spectroscopy, and by the PS one appeared to be consistent with each other as well as with the results of the theoretical calculations reported in the literature (Stier et al., 1999).

\section{InGaAs/GaAs(001) quantum rings}

\subsection{Growth and characterization}

In this subsection, the details on the growth of the InAsGa/GaAs(001) QRs by AP MOVPE as well as of their characterization are presented.

The procedure of growing the self-assembled InAsGa/GaAs(001) QRs by AP MOVPE was different from that used in the standard MBE. Usually, in order to grow the InGaAs/GaAs(001) QRs by standard MBE, the InAs/GaAs(001) QDs are capped by a thin GaAs cladding layer with $d_{\mathrm{c}} \approx\langle h\rangle$, and then the structures are annealed at the temperature $T_{\mathrm{A}} \approx 600 \div 630^{\circ} \mathrm{C}$ for several tens of minutes (Lorke et al., 2002). However, the QRs grown by AP MOVPE have been proven to form just during capping of the InAs QDs by the GaAs cladding layer (Baidus' et al., 2000). This phenomenon had been attributed to the enhanced surface diffusion of In adatoms in the AP MOVPE process as compared to MBE because in the former case the surface dangling bonds are passivated by hydrogen residual from the cracking of the metal organic compounds and/or arsine.

The InGaAs/GaAs(001) QR structures for the Tunnelling AFM investigations described in the present chapter have been grown on the $n^{+}-\mathrm{GaAs}(001)$ substrates misoriented by $5^{\circ}$ towards $<110>$. 

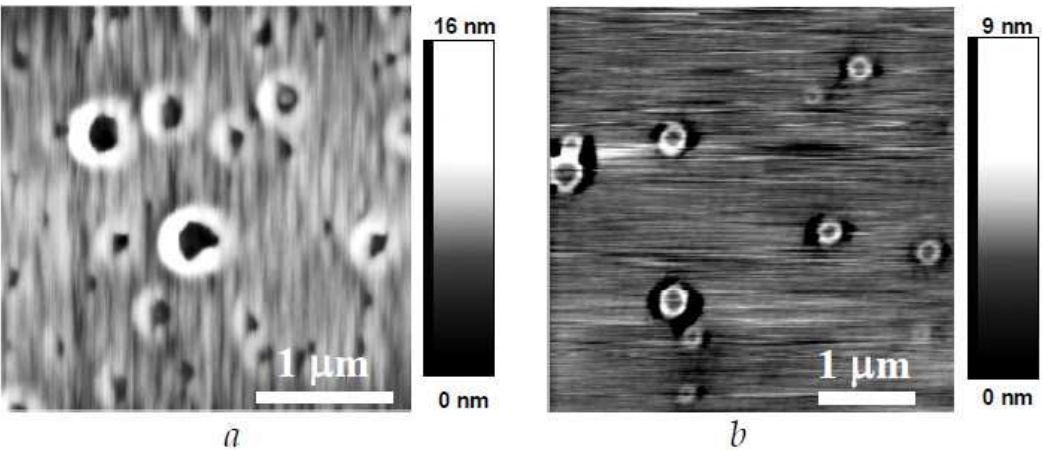

Fig. 11. The ambient air AFM image of an InGaAs/GaAs(001) QR structure (a); a liquid AFM image of an InGaAs/GaAs(001) QR structure with a $30 \mathrm{~nm}$ thick GaAs cladding layer removed by selective wet etching. Reproduced from (Filatov et al., 2011) with permission from $\odot$ Pleiades Publishing, Ltd.

The $3 \mathrm{~nm}$ thick intentionally undoped GaAs spacer layers have been grown on the $n^{+}$-GaAs buffer layers. Then, the InAs/GaAs QDs were grown at $T_{\mathrm{g}}=530^{\circ} \mathrm{C}$ and capped by a GaAs cladding layer with the thickness $d_{\mathrm{c}}=10 \mathrm{~nm}$ at $T_{\mathrm{g}}=600^{\circ} \mathrm{C}$. The nominal thickness of the InAs layer $d_{\text {InAs }}$ was $\approx 5 \mathrm{ML}$. The ambient air AFM measurements have demonstrated the InGaAs QRs to form during capping the InAs QDs at elevated temperatures (Fig. 11, a). The QRs of various diameters $D=150 \div 400 \mathrm{~nm}$ have been observed on surface (Fig. 11, a). As it had been found earlier (Karpovich et al., 2004b), the structures with the surface InAs/GaAs(001) QDs grown by AP MOVPE are features by a number of the relaxed InGaAs clusters present on the surface (Fig. 12,b). These clusters are formed via the coalescence of the smaller InAs coherent QDs during growth, which, in turn, has been attributed to the enhanced surface diffusion in the AP MOVPE process. Within this paradigm, the formation of the smaller QRs has been attributed to the transformation of the smaller coherent InAs/GaAs QDs during capping while the formation of the larger QRs has been attributed to the transformation of the larger relaxed InGaAs clusters.

Also, the structure with $d_{\mathrm{c}} \approx 30 \mathrm{~nm}$ was grown on a semi insulating $\mathrm{GaAs}(001)$ substrate in order to examine the optical properties of the QRs by PL spectroscopy. In order to reveal the morphology of the overgrown QRs, we have employed the removal of the GaAs cladding layer by wet selective etching followed by AFM investigation in liquid (Karpovich et al., 2004a). The application of liquid AFM was to avoid the probe fadeouts due to the residual etching solution contamination (Fig. 11, b).

The PL $(77 \mathrm{~K})$ and PS (300 K) spectra of the structures with the thickness of the GaAs cladding layer $d_{\mathrm{c}} \approx 30$ and $10 \mathrm{~nm}$, respectively, are presented in Fig. 12, $a$. A peak of the edge PL of GaAs with the maximum energy $h v_{\mathrm{m}} \approx 1.51 \mathrm{eV}$ (GaAs) and a peak with $h v_{\mathrm{m}} \approx 1.41 \mathrm{eV}$ $(\mathrm{WL})$ originating from the interband radiative transitions between the ground quantum confined states in the InAs/GaAs(001) wetting layer are present in the PL spectrum. Also, a broad PL band with $h v_{\mathrm{m}} \approx 1.2 \mathrm{eV}$ attributed to the ground state transitions in the InGaAs/GaAs(001) QRs have been observed. This band has a longer wavelength shoulder and can be decomposed into two Gaussian components with $h v_{\mathrm{m}} \approx 1.2$ and $h v_{\mathrm{m}} \approx 1.1 \mathrm{eV}$ related to the smaller and larger QRs, respectively. 


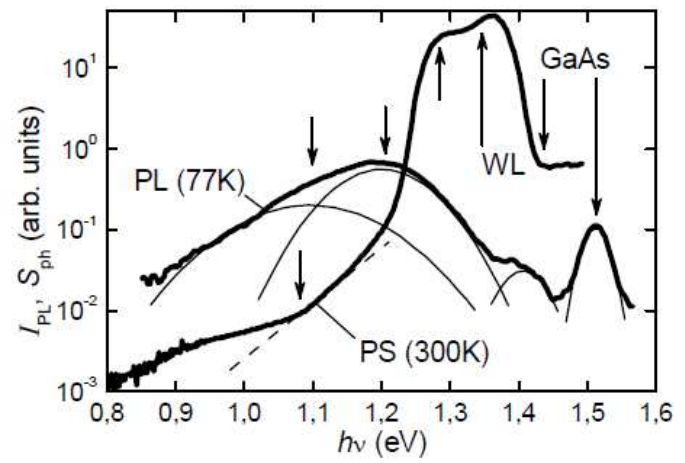

a

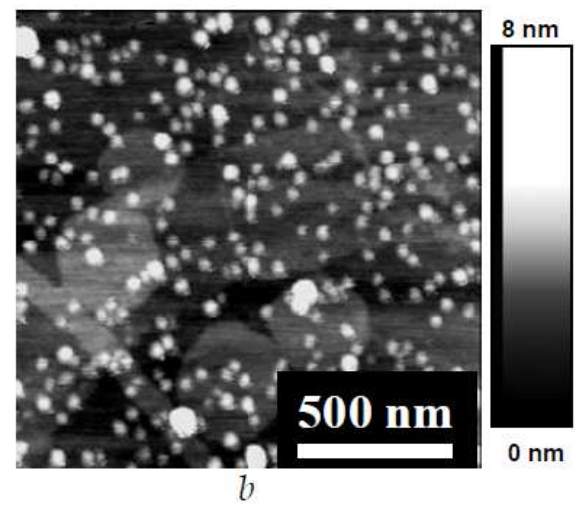

Fig. 12. The PL (77 K) and photosensitivity (300K) spectra of the InGaAs/GaAs(001) QR structures with the $30 \mathrm{~nm}$ and $10 \mathrm{~nm}$ cladding layers, respectively $(a)$. Reproduced from (Filatov et al., 2011) with permission from OPleiades Publishing Ltd.; an AFM image of a surface InAs/GaAs(001) QDs (b).

Also, the PS bands with the edges at $h v \approx 1.36 \mathrm{eV}(\mathrm{WL}), 1.29 \mathrm{eV}$, and $1.09 \mathrm{eV}$ corresponding to the above PL peaks have been observed in the PS spectrum (Fig. 12,a). The difference in the values of the PL peak energies and the band edges ones in the PS spectrum could be related to the different in $d_{c}$, and, in turn, to the difference in the morphology, in the composition, and in the elastic strain of the $\mathrm{QR}$ material in these structures. Note that since the structure was photoexcited through the substrate, playing a role of a low-pass optical filter, the PS spectrum was truncated at $h v \approx 1.43 \mathrm{eV}$ that is the optical gap energy of $n^{+}-$ GaAs. The PS band with the edge at $h v \approx 0.8 \mathrm{eV}$ was related, most likely, to the impurity PS from the defect complexes in GaAs (Karpovich et al., 2004b).

The In molar fraction $x$ in the QR material $\left(\operatorname{In}_{x} \mathrm{Ga}_{1-x} \mathrm{As}\right)$ was estimated from the PL and PS spectra to be $0.35 \div 0.4$. The estimates were made by the best fit between the energies of the PL peak and of the PS band edges related to the ground state transitions in the QRs and the calculated values of the ground state transitions in the QRs $E_{0}, x$ being the fitting parameter. The values of $E_{0}$ as a function of the QRs' sizes determined form the AFM images and of $x$ were calculated by solving the Schrödinger's equation in the effective mass approximation for a model potential of a flat gasket-shaped ring (rectangular in the cross-section) with potential wells of finite height (Barticevic et al., 2000). The ring material was assumed to be pseudomorphic to the GaAs matrix. The materials parameters (the effective masses of electron and holes masses, the band offsets, etc.) were taken from (Stier et al., 1999).

\subsection{Tunneling AFM investigations}

In this subsection, the results of the investigations of the electronic structure of the self assembled InGaAs/GaAs(001) QRs by Tunnelling AFM are presented. Again, it is worth noting that the authors of the present chapter had applied Scanning Probe Microscopy technique for the studying of the LDOS in the self assembled semiconductor QRs for the first time. 

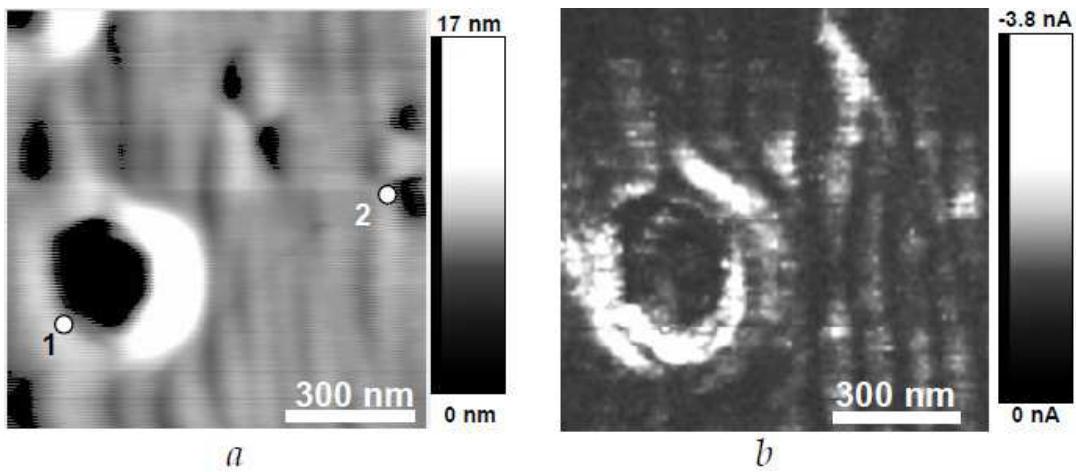

Fig. 13. The UHV AFM $(a)$ and the probe current $(b)$ images of an InGaAs/GaAs(001) QR structure. $V_{\mathrm{g}}=-1.37 \mathrm{~V}$. The figures (1) and (2) mark the points of measurement of the $I-V$ curves (the respective $\sigma_{\mathrm{d}}\left(V_{\mathrm{g}}\right)$ spectra are presented in Fig. 14). Reprinted from (Filatov et al., 2010) under license by IoP Publishing Ltd.

The UHV AFM and the probe current images of an InGaAs/GaAs(001) QR structure are presented in Fig. 13. The QRs are manifested in the probe current image $I_{t}(x, y)$ by the increased probe current $I_{\mathrm{t}}$ that was related to the tunnelling of the electrons from the probe coating material into the $n^{+}$-GaAs buffer through the quantum confined states in the InGaAs/GaAs(001) QRs (Fig 4,b). The differential conductivity spectra $\sigma_{\mathrm{d}}\left(V_{\mathrm{g}}\right)$ of the contact of a Pt coated AFM tip to the surface of the InGaAs QRs on the $n$-GaAs $/ n^{+}-\mathrm{GaAs}(001)$ substrate are presented in Fig. 14. The peaks related to the quantum confined states in the QRs are well expressed in the $\sigma_{\mathrm{d}}\left(V_{\mathrm{g}}\right)$ spectrum of the smaller QR (Fig. 14, $b$ ). However, the features, which could be related to the quantum size effect are present in the $\sigma_{\mathrm{d}}\left(V_{\mathrm{g}}\right)$ spectrum of the larger QR as well (Fig. 14,a). So far, the larger QRs could be classified as the quantum size structures as well in spite of their relatively large sizes. The calculations have shown that the size quantization in $z$ direction (normal to the substrate) affects the energy spectrum of the electrons and holes in QRs most.
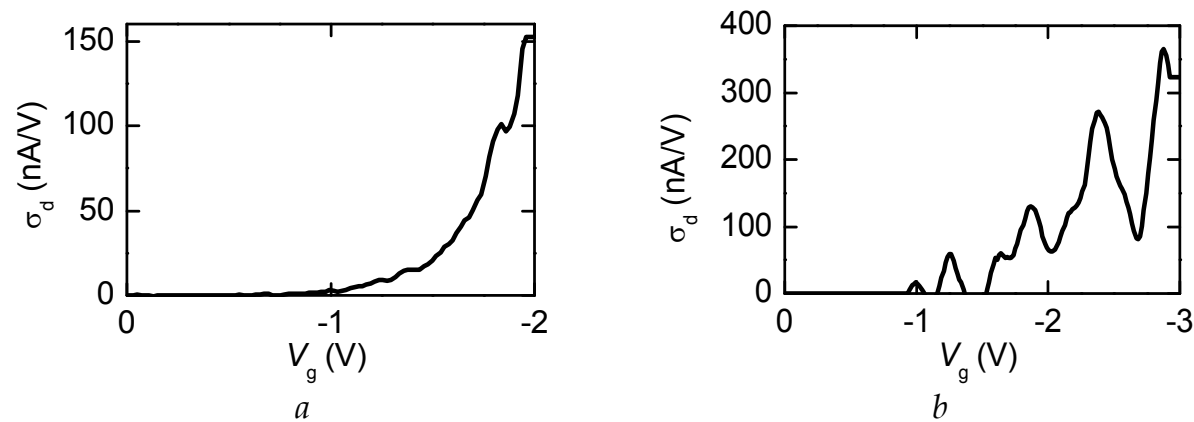

Fig. 14. The differential conductivity spectra $\sigma_{\mathrm{d}}\left(V_{\mathrm{g}}\right)$ of the contact of a Pt coated AFM tip to the surface of the InGaAs QRs of larger $(a)$ and smaller $(b)$ size on the $n$-GaAs $/ n^{+}-\mathrm{GaAs}$ (001) substrate. The points of measurement of the initial $I-V$ curves are marked in Fig. 13, a. Reproduced from (Filatov et al., 2011) with permission from @Pleiades Publishing, Ltd. 


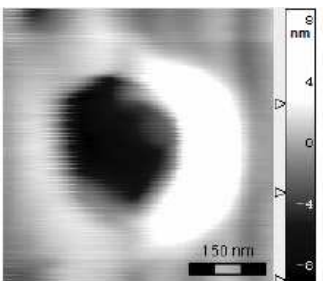

a

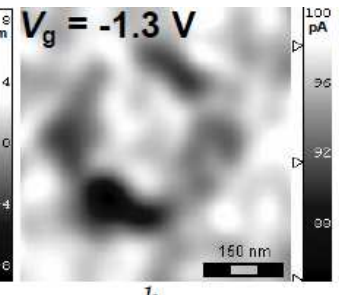

$b$

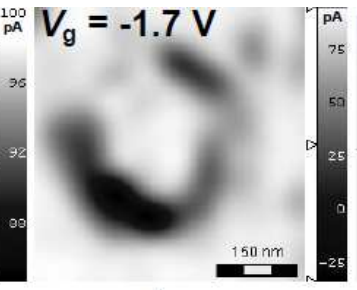

C

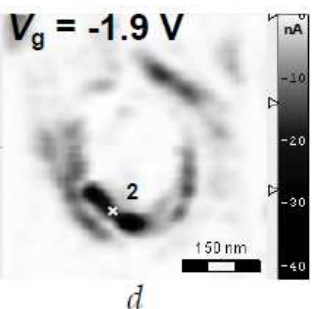

Fig. 15. The AFM $(a)$ and the inverted probe current $(b-d)$ images of an InGaAs QR on the $n$-GaAs $/ n^{+}-\mathrm{GaAs}(001)$ substrate. Reproduced partly from (Filatov et al., 2011) with permission from OPleiades Publishing, Ltd.

The AFM images and the inverted probe current images of an individual InGaAs/GaAs(001) QR measured at different values of $V_{\mathrm{g}}$ are presented in Fig. 15. The probe current images of the QR change with increasing $V_{\mathrm{g}}$. The most important result of the studies presented in this section was the observation of the angular patterning in the probe current images of the QRs. Ideally, in the circularly symmetric potential (Curie group of symmetry $C_{\infty}$ ), the angular dependence of the envelope eigenfuntions of the quantum confined states is expressed in the polar coordinates $(r, \varphi)$ by the term $\exp (-i l \varphi)$ where $i$ is the imaginary unity and $l=0, \pm 1, \ldots$ is the angular quantum number. As $|\exp (-i l \varphi)|^{2}=1$ for any $l$ and $\varphi$, no angular dependence of $I_{\mathrm{t}}$ should be observed.

The observation of the angular patterning in the current images of the QRs has been attributed to the asymmetry of the QRs' shape. In addition, one should take into account the effect of the piezoelectric field in the strained InGaAs, which also reduces the potential symmetry from $C_{\infty}$ downto $C_{2 v}$ even in a perfectly round $Q R$ (Stier et al., 1999).

In order to account for the effect of the effect of the strain-induced piezoelectric field on the angular pattern of the LDOS in the InGaAs/GaAs(001) QRs, we have applied a simple model in the framework of the first-order perturbation theory. The lateral part of the spinindependent eigenfunction for the model flat gasket finite well height potential (Barticevic et al., 2000) can be written in the form:

$$
\chi_{\mathrm{ml}}(\mathrm{r}, \varphi)=\chi_{\mathrm{ml}}(\mathrm{r}) \exp (-\mathrm{il} \varphi),
$$

where $\chi_{m l}(r)$ is the radial part of $\chi_{m l}(r, \varphi)$ expressed via the Bessel functions and $m$ is the radial quantum number. As the perturbation piezoelectric potential has the $C_{2 v}$ symmetry, an additional integral of motion, parity of states, appears. So, one can write the angular components of the correct envelopes of the zero-order approximation as

$$
\begin{aligned}
& \chi_{0}^{(0)}(\varphi)=\frac{1}{\sqrt{2 \pi}} \\
& \chi_{\mu+}^{(0)}(\varphi)=\frac{1}{\sqrt{\pi}} \cos (\mu \varphi), \\
& \chi_{\mu-}^{(0)}(\varphi)=\frac{1}{\sqrt{\pi}} \sin (\mu \varphi)
\end{aligned}
$$

where $\mu=|l|=1,2, \ldots,|m-1|$. 


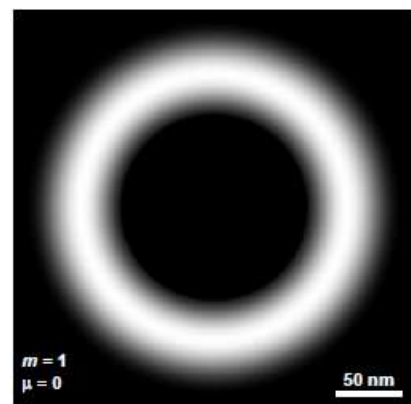

$a$

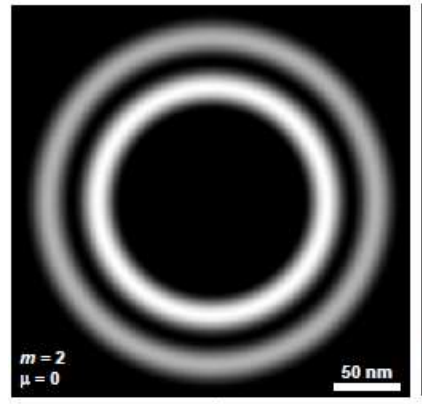

$d$

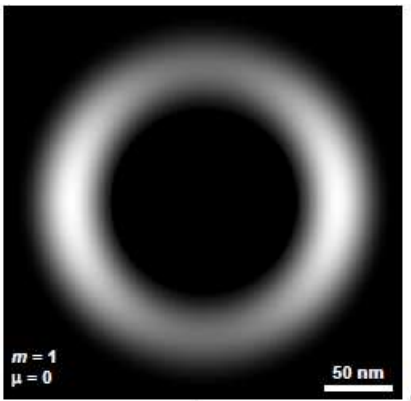

$b$

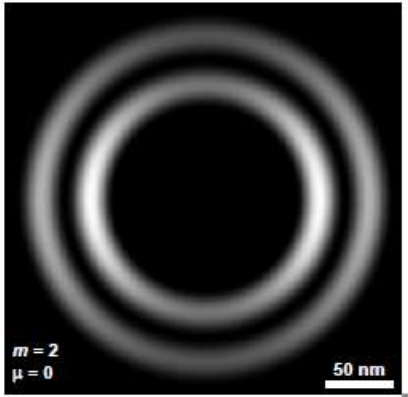

e

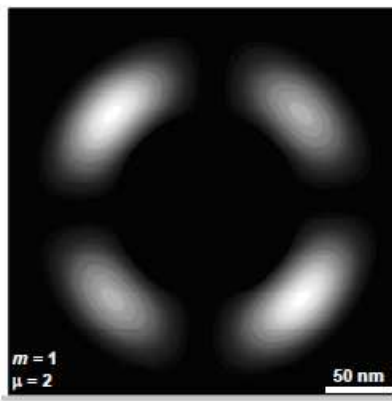

$c$

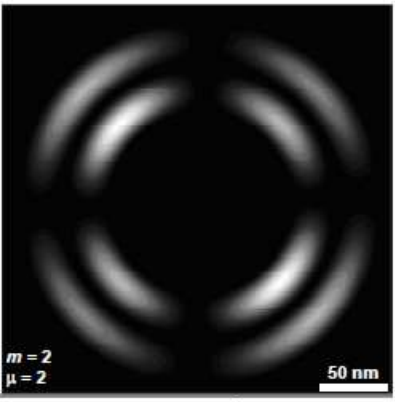

$f$

Fig. 16. The model maps of the spatial distribution of the lateral component of the probability density of the envelope wavefunction of the quantum confined states $\left|\chi_{n l}(r, \varphi)\right|^{2}$ in the plane of an $\operatorname{In}_{0.35} \mathrm{Ga}_{0.65} \mathrm{As} / \mathrm{GaAs}(001)$ for different values of $m$ and $\mu$. $(a, d)$ : no perturbation potential; $(b, c, e, f)$ : the perturbation potential $V_{0} \cos (2 \varphi)$ is imposed. Reproduced from (Filatov et al., 2011) with permission from (OPleiades Publishing Ltd.

The model perturbation potential to account for the effect of the piezoelectric field has been selected in the form $V(\varphi)=V_{0} \cos (2 \varphi)$. Such a model potential satisfies the symmetry $C_{2 v}$ and is convenient for the calculations of the matrix elements which appeared to be equal to zero except the $<m l \mid m l \pm 2>$ ones.

The model maps of $\left|\chi_{n l}(r, \varphi)\right|^{2}$ calculated for the $\operatorname{In}_{0.35} \mathrm{Ga}_{0.65} \mathrm{As} / \mathrm{GaAs}(001)$ QRs with the outer and the inner radii of 70 and $140 \mathrm{~nm}$, respectively, are presented in Fig. 16. When no perturbation potential is imposed, no angular patterning of the probability density maps takes place (Fig. 16, $a \& d$ ). However, the model $\left|\chi_{n l}(r, \varphi)\right|^{2}$ maps calculated in the first order of the perturbation theory for $V_{0}=5 \mathrm{meV}$ demonstrate well expressed angular patterning. Note that the probability density images keep the $C_{2 v}$ symmetry regardless to the number of knots in the circle, in accordance with Curie's theorem.

Comparing the calculated $\left|\chi_{n l}(r, \varphi)\right|^{2}$ patterns with the the experimental probe current images of the QRs (cf, for example, Fig. 16, c \& Fig. 15, b; Fig. 16, $f$ \& Fig. 15, d) demonstrate that in general the model presented above describes the qualitative features of the experimental current images more or less accurately. In turn, this could be considered as an evidence for the asymmetry of the quantum confining potential in the QRs as the origin of the angular patterning of these ones. 


\section{GeSi/Si(001) nanoislands}

\subsection{Growth and characterization}

In this section, the results of the investigation of the LDOS in the self-assembled $\mathrm{GeSi} / \mathrm{Si}(001)$ self assembled nanoislands by Tunnelling AFM are presented. Again, it should be stressed here that the authors of the present chapter had applied Scamming Probe Microscopy to the investigation of the LDOS in the self-assembled GeSi/Si(001) nanoislands for the first time. (Borodin et al., 2011).

The heterostructures with the surface $\mathrm{GeSi} / \mathrm{Si}(001)$ nanoislands for the Tunnelling AFM investigations had been grown on the $p^{+}-\mathrm{Si}(001)$ substrates by $\mathrm{SMBE}$ in $\mathrm{GeH}_{4}$ ambient using a home-made UHV setup (Svetlov et al., 2001). The qualitative band diagrams of the structures for the Tunnelling AFM investigations are presented in Fig. 19, $b \& c$. The buffer layers of $\approx 200 \mathrm{~nm}$ in thickness were doped heavily by boron up to the hole concentration $10^{18} \mathrm{~cm}^{-3}$. More details on the growth technique can be found elsewhere (Filatov et al., 2008a, 2008b).

The topography of the samples destined to the Tunnelling AFM investigations was examined first by ambient air AFM. The AFM images of the structures with the surface $\mathrm{GeSi} / \mathrm{Si}(001)$ nanoislands grown at various growth temperatures $T_{\mathrm{g}}$ are presented in Fig. 17. A dense array of the pyramid-shaped nanoislands $\left(N_{\mathrm{s}} \sim 10^{11} \mathrm{~cm}^{-2}\right)$ has been observed on the surface of the sample grown at $T_{\mathrm{g}}=500^{\circ} \mathrm{C}$ (Fig. 17, a). The (105) faceting of the pyramid nanoislands was resolved poorly, again, due to the convolution effect. A bimodal size distribution of the nanoislands took place on the surface of the sample grown at $T_{\mathrm{g}}=$ $600^{\circ} \mathrm{C}$ (Fig. 17, b). Along with the dome-shaped GeSi islands, a small number of larger so called super dome islands (Kamins et al., 1999) has been observed on the surface. The super dome islands originate from the coalescence of the smaller dome ones. It should be noted that in the structures grown by MBE the formation of the super dome islands has been observed in the process of the post-growth annealing (Kamins et al, 1999). In contrary, in SMBE in $\mathrm{GeH}_{4}$ ambient the formation of the super dome islands has been observed just during growth (Filatov et al., 2008a, 2008b), that was attributed to Ostwald ripening.

The surface density of the islands $N_{\mathrm{s}}$ decreased and their sizes increased with increasing $T_{g}$ while the bimodal size distribution remained in the structures grown at $T_{\mathrm{g}}=700^{\circ} \mathrm{C}$ and $800^{\circ} \mathrm{C}$ (Fig. 17, $c \& d$ ), in accordance with Lifshits-Sloyzov-Wagner theory.

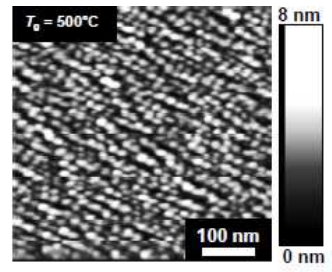

$a$

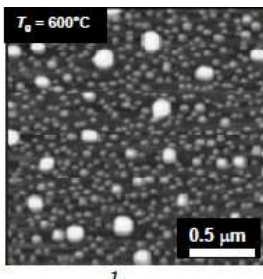

b

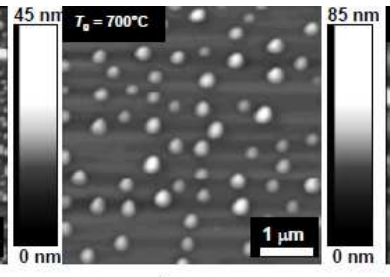

$c$

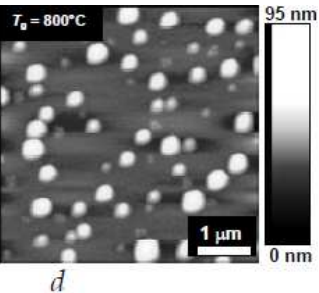

d

Fig. 17. The ambient air AFM images of the surface $\mathrm{GeSi} / \mathrm{Si}(001)$ nanoislands grown by $\mathrm{SMBE}$ in $\mathrm{GeH}_{4}$ ambient at various growth temperatures $T_{\mathrm{g}}$. 

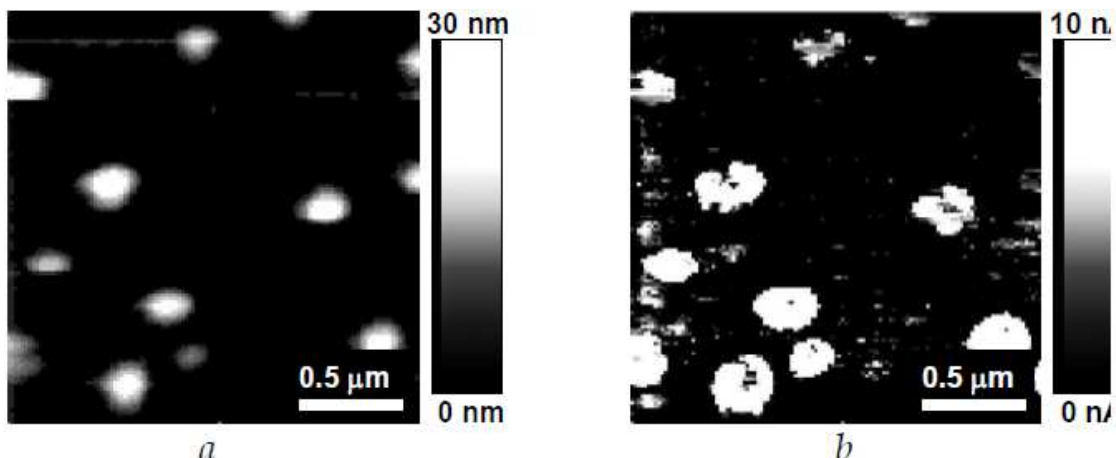

Fig. 18. The UHV AFM (a) and the probe current $(b)$ images of the GeSi/Si(001) nanoislands. $V_{\mathrm{g}}=2.0 \mathrm{~V}$. Reproduced from (Borodin et al., 2011) with permission from @Pleiades Publishing, Ltd.

The composition of the GeSi/Si(001) nanoislands' material has been examined by PL spectroscopy (Filatov et al., 2008a, 2008b) and by Confocal Raman Microscopy (CRM, Mashin et al., 2010). The $\mathrm{GeSi} / \mathrm{Si}(001)$ structures grown on the $p$-Si substrates with the nanoislands grown in the same conditions as the surface ones destined to the Tunnelling AFM studies but capped with the $40 \mathrm{~nm}$ thick cladding Si layers had been used for the optical investigations. It has been found that the nanoislands grown within $T_{\mathrm{g}}=600 \div 800^{\circ} \mathrm{C}$ consisted of $\mathrm{Ge}_{x} \mathrm{Si}_{1-x}$ alloy with the Ge molar fraction $x$ decreasing from $\approx 0.55$ downto $\approx 0.25$ with $T_{\mathrm{g}}$ increasing from $600^{\circ} \mathrm{C}$ up to $800^{\circ} \mathrm{C}$ (Filatov et al., 2008b, Mashin et al., 2010).

\subsection{Tunneling AFM investigations}

The UHV AFM and the probe current images of the surface GeSi/Si(001) nanoislands grown at $T_{\mathrm{g}}=700^{\circ} \mathrm{C}$ are presented in Fig. 18, $a$ and $b$, respectively. The GeSi islands of various sizes and shapes have been observed on the sample surface (Fig. 18, a). The smaller islands were dome shaped while the larger ones (super dome islands) were shaped as the truncated pyramids defined by the (101) facets.

The spots of increased probe current $I_{\mathrm{t}}$ in the probe current image (Fig. 18, $b$ ) were located at the places corresponding to $\mathrm{GeSi}$ islands. The increased probe current has been related to the tunnelling of the electrons from the electronic states in the valence band of the GeSi islands into the free states above the Fermi level $E_{\mathrm{F}}$ in the Pt AFM tip coating through the native oxide layer covering the island surface. The band diagram of a positively biased contact between the Pt coated AFM tip and a surface GeSi $/ p-\mathrm{Si} / p+-\mathrm{Si}$ island is shown in Fig. $19, c$. It should be noted that the sizes of the spots of increased $I_{t}$ in the probe current image (Fig. 18, b) are larger than the sizes of the topographic images of the respective islands in Fig. 18, $a$. This has been attributed to the convolution effect due to the relatively large radius of the curvature of the AFM tip $R_{\mathrm{p}}$. Typical values of $R_{\mathrm{p}}$ for NT MDT® NSG-01 Pt coated AFM probes are $\approx 35 \mathrm{~nm}$, according to the vendor's specifications.

The differential conductivity spectra $\sigma_{\mathrm{d}}\left(V_{\mathrm{g}}\right)$ of the contact of a Pt coated AFM tip to the structure with the surface pyramid $\mathrm{Ge} / \mathrm{Si}(001)$ nanoislands grown at $T_{\mathrm{g}}=500^{\circ} \mathrm{C}$ are presented in Fig. 19, $a$. 


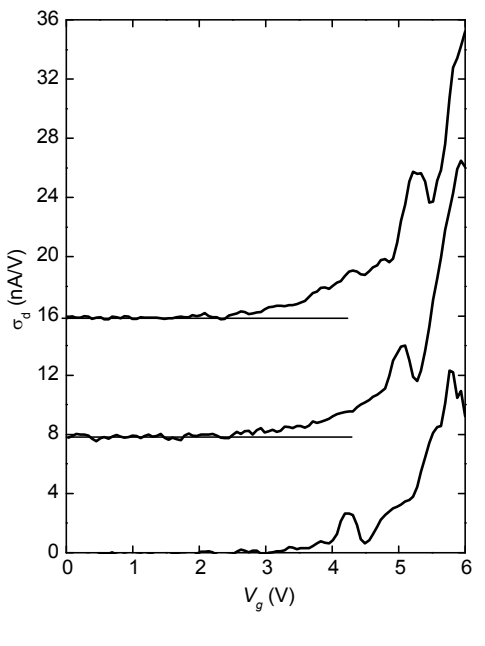

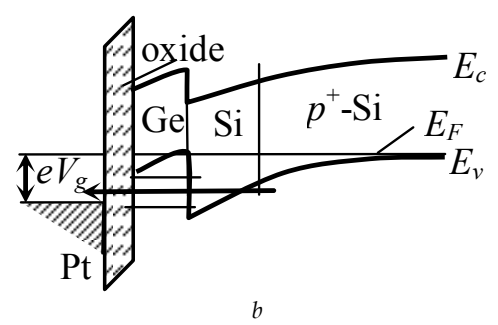

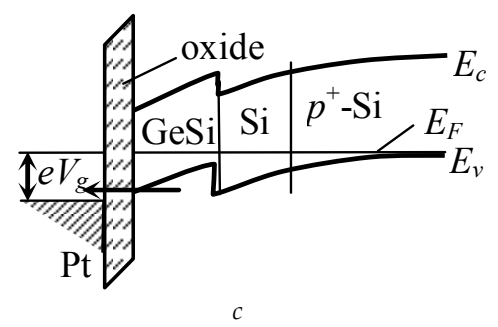

Fig. 19. The differential conductivity spectra $\sigma_{\mathrm{d}}\left(V_{\mathrm{g}}\right)$ of the contact of a Pt coated AFM tip to the structure with the surface pyramid Ge/Si(001) nanoislands grown at $T_{\mathrm{g}}=500^{\circ} \mathrm{C}(\mathrm{a})$, the qualitative band diagrams of the positively biased contact s of the Pt coated AFM tip to the pyramid islands $(b)$ and to the dome-shaped or the super-dome islands $(c)$.

Again, as in the cases of the InAs/GaAs(001) QDs and of the InGaAs/GaAs(001) QRs (see Sec. $2 \& 3$ above), the differential conductivity spectra $\sigma_{\mathrm{d}}\left(V_{\mathrm{g}}\right)$ have been calculated from the measured $I_{\mathrm{t}}\left(V_{\mathrm{g}}\right)$ curves by numerical differentiation with non-linear smoothing. The spectra presented in Fig. 19, a were the results of the averaging of $25 I_{\mathrm{t}}\left(V_{\mathrm{g}}\right)$ curves measured in the different areas on the sample surface $5 \times 5$ pixels by size. The peaks in the $\sigma_{\mathrm{d}}\left(V_{\mathrm{g}}\right)$ spectra were ascribed to the tunneling of the electrons from the valence band states in the $p^{+}-\mathrm{Si}$ buffer through the quantum confined hole states in the $\mathrm{Ge} / \mathrm{Si}(001)$ pyramid islands into the free states above the Fermi level in the Pt AFM tip coating (Fig. 19, b).

We have failed to obtain the probe current images, which could be associated to the probability density patterns $\left|\chi(x, y)^{2}\right|$ of the quantum confined hole states in the pyramid $\mathrm{Ge} / \mathrm{Si}(001)$ nanoislands. The most probable cause for this, in our opinion, were too small sizes $(b=50 \div 80$ $\mathrm{nm}$ and $h=5 \div 8 \mathrm{~nm}$ ) and too large surface density $\left(N_{\mathrm{s}} \sim 10^{11} \mathrm{~cm}^{-2}\right)$ of the Ge nanoislands on the surface of this particular sample as compared to the AFM probe tip dimensions $\left(R_{\mathrm{p}} \approx 35 \mathrm{~nm}\right)$. As a result, the islands have been resolved poorly in the UHV AFM images due to the convolution artifact unlike the ambient air AFM (Fig. 17, a) measured with NT MDT® CSG-01 probes without metal coating $\left(R_{\mathrm{p}}<10 \mathrm{~nm}\right.$, according to the vendor's specifications).

The differential conductivity spectra $\sigma_{\mathrm{d}}\left(V_{\mathrm{g}}\right)=d I_{\mathrm{t}} / d V_{\mathrm{g}}$ and the normalized differential conductivity spectra $\left(d I_{\mathrm{t}} / d V_{\mathrm{g}}\right) /\left(I_{\mathrm{t}} / V_{\mathrm{g}}\right)$ of the contact of the Pt coated AFM probe tip to the $\mathrm{GeSi} / \mathrm{Si}(001)$ nanoislands grown at various temperatures and to the sample surface between the islands (WL) are presented in Fig. 20. The points on the sample surface where the initial $I_{\mathrm{t}}\left(V_{\mathrm{g}}\right)$ curves have been measured are marked in Fig. 21. The spectra presented in Fig. 20 were the results of the averaging of $25 I_{\mathrm{t}}\left(V_{\mathrm{g}}\right)$ curves measured in the spots on the sample surface $5 \times 5$ pixels by size. 


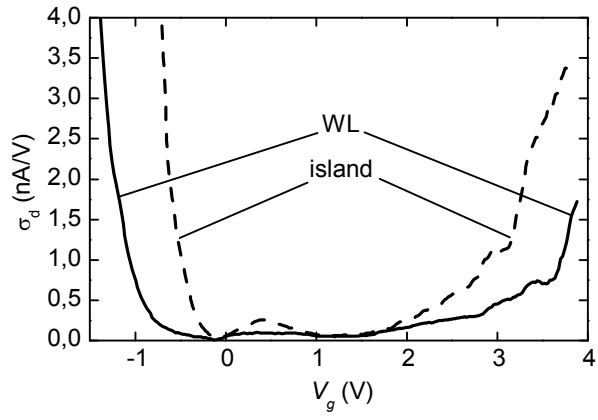

$a$

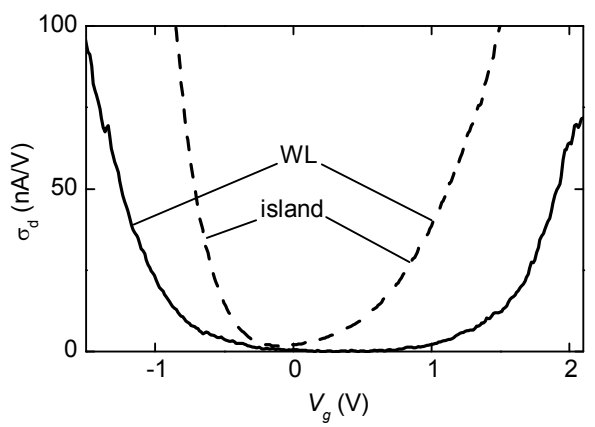

C

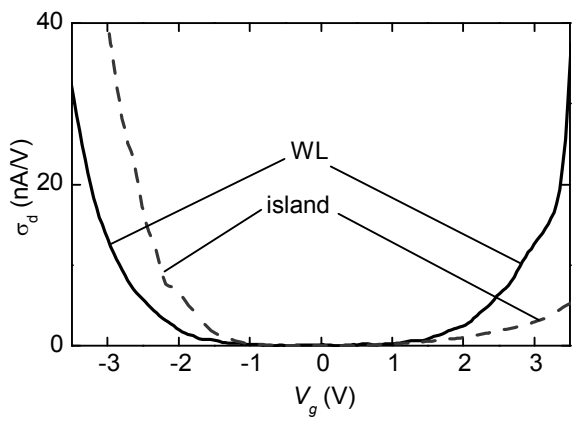

$e$

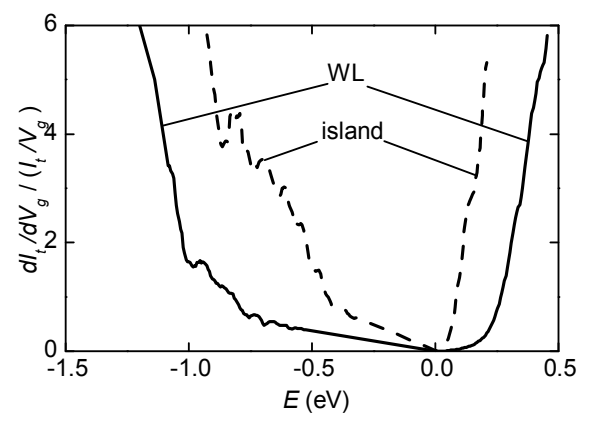

$b$

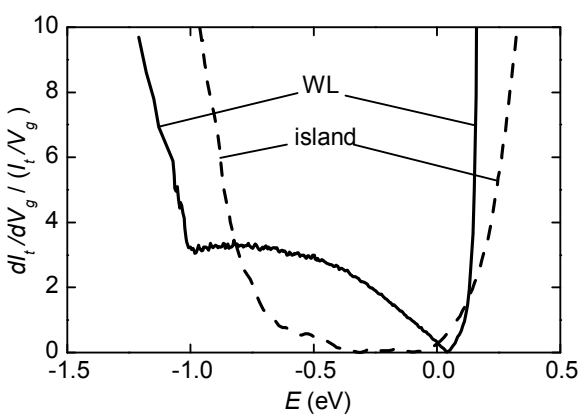

$d$

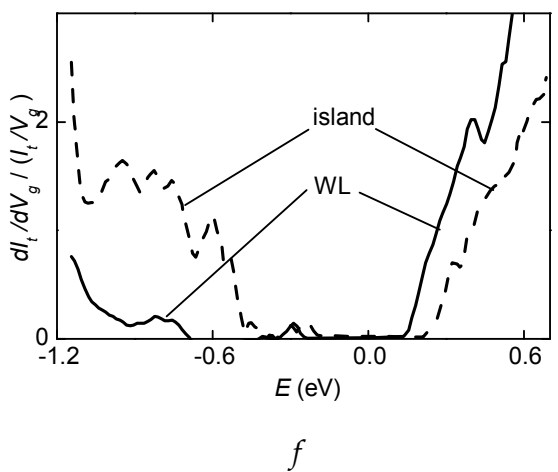

Fig. 20. The differential conductivity spectra $\sigma_{\mathrm{d}}\left(V_{\mathrm{g}}\right)(a, c, e)$ and the normalized differential conductivity spectra $\left(d I_{\mathrm{t}} / d V_{\mathrm{g}}\right) /\left(I_{\mathrm{t}} / V_{\mathrm{g}}\right)(b, d, f)$ of the contact of the Pt coated AFM probe tip to the $\mathrm{GeSi} / \mathrm{Si}(001)$ nanoislands and to the sample surface between the islands (WL). The points on the sample surface where the initial $I_{\mathrm{t}}\left(V_{\mathrm{g}}\right)$ curves have been measured are denoted in Fig. 21. Reproduced partly from (Borodin et al., 2011) with permission from (Pleiades Publishing, Ltd. 


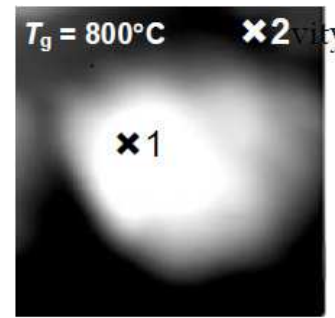

a

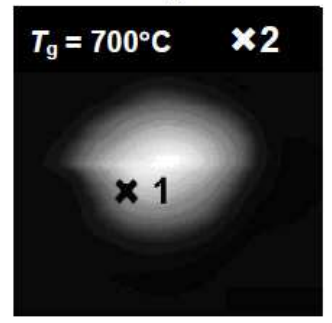

$e$

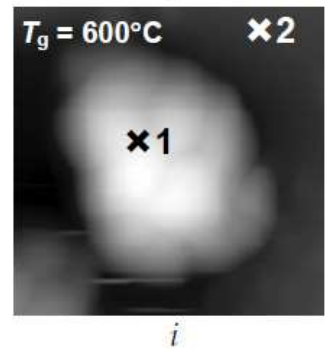

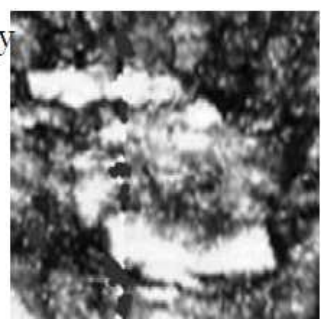

$b$

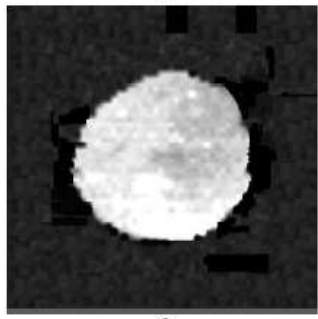

$f$

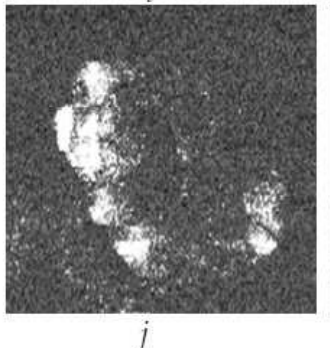

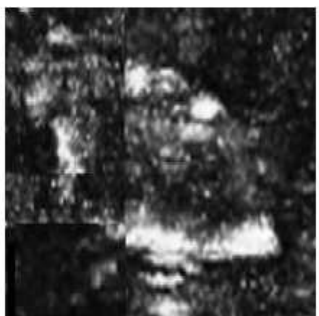

C

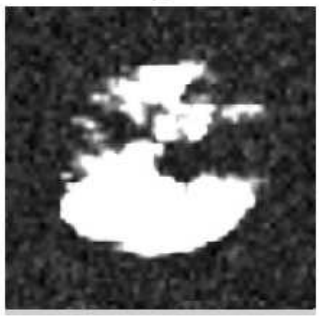

$g$

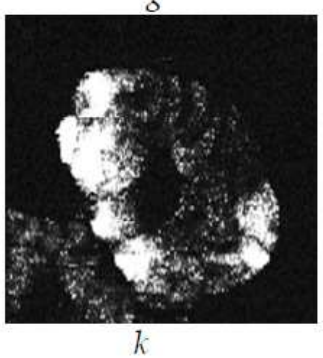

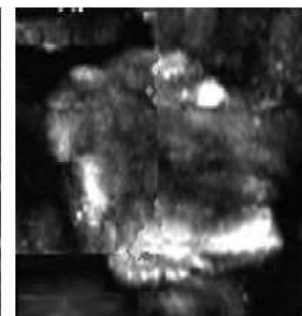

d

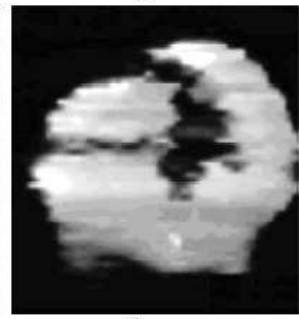

7

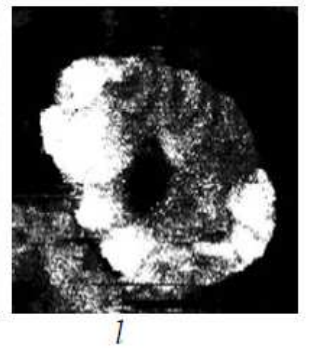

Fig. 21. The AFM $(a, e, i)$ and the runnel current $(b-d, f-h, j-l)$ images of the individual $\mathrm{GeSi} / \mathrm{Si}(001)$ nanoislands grown by SMBE in $\mathrm{GeH}_{4}$ ambient at various growth temperatures $T_{\mathrm{g}}$. The points of measurements of the $I_{\mathrm{t}}\left(V_{\mathrm{g}}\right)$ curves the derivatives of which are presented in Fig. 20 are denoted in the AFM images $(a, e, i)$. Reproduced partly from (Borodin et al., 2011) with permission from $\mathrm{OPleiades}$ Publishing, Ltd.

According to the theory of the tunnel spectroscopy, the normalized differential conductivity spectra $\left(d I_{\mathrm{t}} / d V_{\mathrm{g}}\right) /\left(I_{\mathrm{t}} / V_{\mathrm{g}}\right)$ of the contact of a metal STM tip to a sample is proportional to the LDOS at the sample surface. The relationship of the energies in the LDOS spectra to $V_{\mathrm{g}}$ again, has been established taking into account the partial drop of $V_{\mathrm{g}}$ on the depletion layer of the tip-to-sample contact on the base of one-dimensional Poisson's equation (Feenstra \& Stroscio, 1987). Unlike the case of the InAs/GaAs(001) QDs considered above in Sec. 2, the charge on the surface states on the boundary of the semiconductor with the native oxide has been neglected because the $\mathrm{SiO}_{2} / \mathrm{Si}$ and $\mathrm{GeO}_{2} / \mathrm{Ge}$ interfaces are known to be featured by low density of the surface states.

As it is evident from Fig. 20, tunnel spectra of the GeSi/Si(001) nanoislands grown at $T_{\mathrm{g}}=$ 700 and $800^{\circ} \mathrm{C}$ demonstrate the I-type conduction band alignment. Traditionally, the $\mathrm{GeSi} / \mathrm{Si}(001)$ heterostructures had been being considered to be the II-type ones, i. e. the GeSi layer had been being treated as a potential barrier for the electrons with respect to the ones 
in Si (Berbezier \& Ronda, 2009). However, there has been an increasing number of publications, both theoretical and experimental, where the $\mathrm{Ge}_{x} \mathrm{Si}_{1-x} / \mathrm{Si}(001)$ heterostructures have been reported to be of the I type ones at low enough values of $x$, i. e. the GeSi layers were proven to be the potential wells for the electrons. More recent 30-band $k \cdot p$ calculations (El Kurdi et al., 2006) have demonstrated the pseudomorphic $\mathrm{Ge}_{x} \mathrm{Si}_{1-x} / \mathrm{Si}(001)$ heterolayers to be the ones of the I type when $0.05<x<0.45$. In contrary, the GeSi/Si(001) nanoislands were reported to be always the II type heterostructures within the whole range of $0<x<1$ due to the nonuniform tensile strain of Si near the tops and the bottoms of the islands (El Kurdi et al., 2006). Anyway, the magnitude of the conduction band offset $\Delta E_{\mathrm{c}}$ do not exceed several meV.

The $I_{t}(x, y)$ images of the GeSi/Si(001) islands were found to depend on $V_{\mathrm{g}}$. At lower $V_{\mathrm{g}}$ corresponding to the extraction of the electrons From the electron states near the top of the valence band in the GeSi islands (see Fig. 19, $b$ ), the probe current images had more or less round shape (Fig. 21, $b, f, j$ ). At higher $V_{\mathrm{g}}$ the current image patterns transformed into the ones having a twofold-like symmetry Fig. 21, $c, g, k$ ). With further increasing of $V_{\mathrm{g}}$ corresponding to the extraction of the electrons from the electron states near the Si valence band edge, the current images took a 4 -fold-like symmetry Fig. 21, $d, h, l$ ) that was attributed to the elastic strain relaxation at the pyramidal island edges.

The values of $\Delta E_{\mathrm{c}}$ at the $\mathrm{Ge}_{x} \mathrm{Si}_{1-x} / \mathrm{Si}(001)$ nanoislands' heterointerface determined from the tunnel spectra of the individual islands presented in Fig. 20 are plotted $v$ s the averaged Ge molar fraction in the nanoisland material $\langle x\rangle$ determined by PL spectroscopy and CRM (Filatov et al., 2008b, Mashin et al., 2010) in Fig. 22, a. Also, the theoretical dependence of $\Delta E_{\mathrm{c}}$ on $\langle x\rangle$ calculated for a strained $\mathrm{Ge}_{x} \mathrm{Si}_{1-x} / \mathrm{Si}(001)$ heterosturcture at $300 \mathrm{~K}$ according to (Aleshkin \& Bekin, 1997) is presented in Fig. 22, $a$. The theory predicts the strained $\mathrm{Ge}_{x} \mathrm{Si}_{1-}$ ${ }_{x} / \mathrm{Si}(001)$ heterostructures to be the ones of the I type when $0<\mathrm{x}<0.44$, the conduction band minima in the strained GeSi are related to the $\Delta_{4}$ valleys. The experimental data of the tunnel spectroscopy agree qualitatively with the theory by (Aleshkin \& Bekin, 1997) as well as the one by (El Kurdi et al., 2006), although the magnitudes of $\Delta E_{\mathrm{c}}$ at the respective values of $\langle x\rangle$ are larger than the ones predicted by the theory.

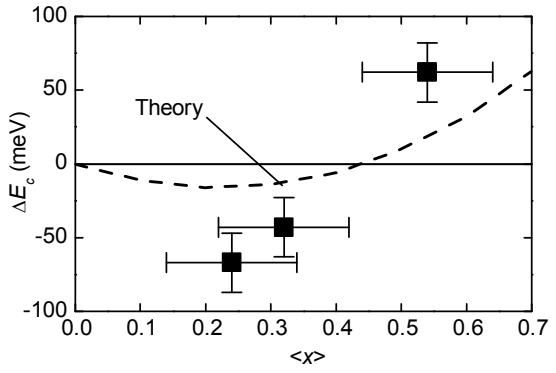

$a$

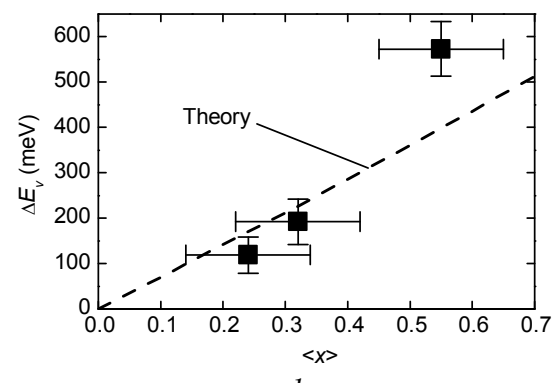

$b$

Fig. 22. The dependencies of the conduction band offset $\Delta E_{\mathrm{c}}(a)$ and of the valence band one $\Delta E_{\mathrm{v}}(b)$ in the surface $\mathrm{Ge}_{x} \mathrm{Si}_{1-x} / \mathrm{Si}(001)$ nanoislands on the averaged Ge molar fraction in the island material $<>$. The theoretical curves have been calculated for the strained $\mathrm{Ge}_{x} \mathrm{Si}_{1-}$ ${ }_{x} / \mathrm{Si}(001)$ layers according to (Aleshkin \& Bekin, 1997). 
This disagreement could be attributed probably to the partial strain relaxation in the surface $\mathrm{GeSi} / \mathrm{Si}(001)$ nanoislands as compared to the pseudomorphic $\mathrm{GeSi} / \mathrm{Si}(001)$ heterostructures or to the coherent $\mathrm{GeSi} / \mathrm{Si}(001)$ nanoislands embedded into the single crystal Si.

Also, the values of the valance band offsets at the $\mathrm{Ge}_{x} \mathrm{Si}_{1-x} / \mathrm{Si}(001)$ nanoislands' heterointerface $\Delta E_{\mathrm{v}}$ determined from the tunnel spectra presented in Fig. 20 are plotted $v \mathrm{~s}$ $<x>$ along with the ones calculated according to (Aleshkin \& Bekin, 1997) in Fig. 22, b. A rather good agreement between the calculated and measured values of $\Delta E_{\mathrm{v}}$ supports the conclusions about the type of the conduction band alignment in the self assembled $\mathrm{GeSi} / \mathrm{Si}(001)$ nanoislands derived out from the tunnel spectroscopy data. Note that unlike the tunnel spectra of the Ge/Si(001) pyramid islands (Fig. 19, a), the tunnel spectra of the larger GeSi/Si(001) islands with lateral sizes $D>100 \mathrm{~nm}$ and height $h>20 \mathrm{~nm}$ (Fig. 20) do not exhibit any features, which could be attributed to the size quantization. This is not surprising taking into account the relatively large sizes of the islands grown at $T_{\mathrm{g}} \geq 600^{\circ} \mathrm{C}$ as compared to the de Broglie wavelength for the holes in GeSi alloy at 300K.

\section{Conclusion}

The results presented in this chapter demonstrate applicability of Tunneling AFM to the ex situ investigations of the LDOS of the quantum confined states in the InGaAs/GaAs and $\mathrm{GeSi} / \mathrm{Si}$ nanostructures covered by the native oxide. The observed patterns of the probe current images agree with the results of the quantum confined states eigenfunction calculations reported in the literature. Besides, the application of Tunnelling AFM technique have brought some fundamental results on the basic properties of various nanostructures. Particularly, the angular patterning of the current images of the InGaAs/GaAs(001) quantum rings was related to the asymmetry of the confining potential. Also, the self assembled $\mathrm{Ge}_{x} \mathrm{Si}_{1-x} / \mathrm{Si}(001)$ nanoislands have been proven to be the I type heterostructures at $x<0.45$.

\section{Acknowledgement}

The authors gratefully acknowledge the financial support from Russian Foundation of Basic Research (RFBR 10-02-90738-mob_st) and from Ministry of Science and Education, Russian Federation (NK-346P-25 and RNP 2.1.1.3615).

\section{References}

Baidus', N.V., Zvonkov, B.N., Filatov, D.O., Guschina, Yu.Yu., Karpovich, I.A. \& Zdoroveischev, A.V. (2000). Investigaton of the overgrowth of the InAs nanoislands in the GaAs/lnAs quantum dot heterostructures grown by Vapor Phase Epitaxy. J. Surf. Investigations: Physics, Chemistry \& Mechanics, No. 7 (July 2000), pp. 71-75, ISSN 1027-4510.

Barticevic, Z., Pacheco, M. \& Latge, A. (2000). Quantum rings under magnetic fields: Electronic and optical properties. Phys. Rev. B, Vol. 62, No. 11 (September, 2000), pp. 6963-6966, ISSN 1098-0121.

Berbezier, I. \& Ronda, A. (2009). SiGe nanostructures. Surf. Sci. Rep., Vol.64, No. 2 (February 2009), pp. 47-98, ISSN 0167-5729. 
Borodin, P.A., Bukharaev, A.A., Filatov, D.O., Isakov, M.A., Shengurov, V.G., Chalkov, V.Yu. \& Denisov, Yu.A. (2011). Investigation of the local density of states in selfassembled $\mathrm{GeSi} / \mathrm{Si}(001)$ nanoislands by Combined Scanning Tunneling and Atomic Force Microscopy. Semicond., Vol.45, No.3 (March 2011), pp.403-407, ISSN 10637826.

Bukharaev, A.A., Berdunov, N.V., Ovchinnikov, D.V. \& Salikhov, K.M. (1998). Threedimensional probe and surface reconstruction for Atomic Force Microscopy using a deconvolution algorithm. Scanning Micros., Vol. 12, No. 1 (January 1998), pp. 225234, ISSN 0891-7035.

El Kurdi, M., Sauvage, S., Fishman, G. \& Boucaud, P. (2006). Band-edge alignment of SiGe/Si quantum wells and SiGeSi self-assembled islands. Phys. Rev. B, Vol. 73, No. 11 (May 2006), p. 195327 (9 p.), ISSN 1098-0121.

Feenstra, R.M. \& Stroscio, J.A. (1987). Tunneling spectroscopy of the GaAs (110) surface. J. Vac. Sci. Technol. B Vol. 5, No. 4 (April 1987), pp. 923-928, ISSN 0734-211X.

Filatov, D.O., Kruglova, M.V., Isakov, M.A., Siprova, S.V., Marychev, M.O., Shengurov, V.G., Chalkov, V.Yu. \& Denisov, S.A. (2008a). Morphology and photoluminescence of self-assembled GeSi/Si nanoclusters grown by Sublimation Molecular-Beam Epitaxy in a $\mathrm{GeH}_{4}$ ambient. Bull. RAS: Physics, Vol. 72, No. 2 (February 2008), pp. 249-252, ISSN 1062-8738.

Filatov, D.O., Kruglova, M.V., Isakov, M.A., Siprova, S.V., Marychev, M.O., Shengurov, V.G., Chalkov, V.Yu. \& Denisov, S.A. (2008b). Photoluminescence of GeSi/Si nanoclusters formed by Sublimation Molecular-Beam Epitaxy in $\mathrm{GeH}_{4}$ medium. Semicond., Vol. 42, No. 9 (September 2008), pp. 1098-1103, ISSN 1063-7826.

Filatov, D.O., Lapshina, M.A., Isakov, M.A., Borodin, P.A. \& Bukharaev, A.A. (2010). Tunnelling AFM study of the local density of states in the self assembled $\mathrm{In}(\mathrm{Ga}) \mathrm{As} / \mathrm{GaAs}(001)$ quantum dots and rings. J. Phys. Conf. Ser. Vol. 245 (October, 2010), p. 012017 (4 p.), ISSN 1742-6596.

Filatov, D.O., Borodin, P.A. \& Bukharaev, A.A. (2011). Study of local density of electron states in InGaAs/GaAs quantum rings by Combined STM/AFM. (2011). J. Surf. Investigation. X-ray, Synchrotron, \& Neutron Techniques, Vol. 5, No. 3 (March 2011), pp. 547-553, ISSN 1027-4510.

Grandidier, B., Niquet, Y. M., Legrand, B., Nys, J. P., Prieste, C., Stievenard, D., Gerard, J.M. \& Thierry-Mieg, V. (2000). Imaging the wave-function amplitudes in cleaved semiconductor quantum boxes. Phys. Rev. Lett., Vol. 85, No. 5 (July 2000), pp. $1068-$ 1073, ISSN 0031-9007.

Hasegawa, H. \& Sawada, T. (1983). On the electrical properties of compound semiconductor interfaces in metaliinsulatorisemiconductor structures and the possible origin of interface states. Thin Solid Films, Vol. 103, No. 1-3 (January 1983), pp. 119-140, ISSN 0040-6090.

Kamins, T.I., Medeiros-Ribeiro, G., Ohlberg, D.A.A. \& Stanley Williams, R. (1999). Evolution of Ge islands on Si(001) during annealing. J. Appl. Phys., Vol. 85, No. 2 (February 1999), pp. 1159-1162, ISSN 1089-7550.

Karpovich, I.A., Zvonkov, B.N., Baidus', N.V., Tikhov, S.V. \& Filatov, D.O. (2004a). Tuning the energy spectrum of the InAs/GaAs quantum dot structures by varying the thickness and composition of a thin double GaAs/InGaAs cladding layer. In:Trends 
in Nanotechnology Research, E.Dirote, pp.173-208, Nova Science, ISBN1-59454-091-8, New York.

Karpovich, I.A., Baidus', N.V., Zvonkov, B.N., Filatov, D.O., Levichev, S.B., Zdoroveishev, A.V. \& Perevoshikov, V.A. (2004b). Investigation of the buried InAs/GaAs quantum dots by SPM combined with selective chemical etching Phys.LowDim.Struct., No. 3/4 (March 2004), pp. 341-345, ISSN 0204-3467.

Lorke, A., Blossey, R., Garcia, J.M., Bichler, M. \& Abstreiter, G. (2002). Morphological transformation of $\mathrm{In}_{y} \mathrm{Ga}_{1-y} \mathrm{As}$ islands, fabricated by Stranski-Krastanov growth. Mater. Sci. Eng. B, Vol. 88, No. 2-3 (January 2002), pp. 225-229, ISSN 0921-5107.

Maltezopoulos, T., Bolz, A., Meyer, C., Heyn, C., Hansen, W., Morgenstern, M. \& Wiesendanger, R. (2003). Wave-function mapping of InAs quantum dots by Scanning Tunneling Spectroscopy. Phys. Rev. Lett., Vol. 91, No. 19 (November 2003), p. 196804 (4 pp.), ISSN 0031-9007.

Mashin, A.I., Nezhdanov, A.V., Filatov, D.O., Isakov, M.A., Shengurov, V.G., Chalkov, V.Yu. \& Denisov, S.A. (2010). Confocal Raman Microscopy of Self-assembled GeSi/Si(001) islands. Semicond., Vol. 44, No. 11 (November 2010), pp. 1504-1510, ISSN 1063-7826.

Medeiros-Ribeiro, G., Bratkovski, A.M., Kamens, T.I., Ohlberg, D.A.A. \& Stanley Williams, R. (1998). Shape transition of germanium nanocrystals on a silicon (001) surface from pyramids to domes. Science, Vol. 279, No. 5349 (January 1998), pp. 353-355, ISSN 1095-9203.

Stier, O., Grundmann, M. \& Bimberg, D. (1999). Electronic and optical properties of strained quantum dots modeled by 8-band $k \cdot p$ theory. Phys. Rev. B, Vol. 59, No. 8 (February 1999), pp. 5688-5701, ISSN 1098-0121.

Suzuki, K., Kanisawa, K., Janer, C., Perraud, S., Takashina, K., Fujisawa, T. \& Hirayama, Y. (2007). Spatial imaging of two-dimensional electronic states in semiconductor quantum wells. Phys.Rev.Lett., Vol.98, No.13 (March 2007), p.136802 (4 p.), ISSN 0031-9007.

Svetlov, S.P., Shengurov, V.G., Tolomasov, V.A., Gorshenin, G.N. \& Chalkov, V.Yu. (2001). A sublimation silicon Molecular Beam Epitaxy system. Instrum. Exp. Tech., Vol. 44, No. 5 (May 2001), pp. 700-703, ISSN 1608-3180.

Yanev, V.; Rommel, M.; Lemberger, M.; Petersen, S.; Amon, B.; Erlbacher, T.; Bauer, A.J.; Ryssel, H.; Paskaleva, A.; Weinreich, W.; Fachmann, C.; Heitmann, J. \& Schroeder, U. (2008). Tunneling atomic-force microscopy as a highly sensitive mapping tool for the characterization of film morphology in thin high- $k$ dielectrics. Appl. Phys. Lett., Vol. 92, No. 25 (June 2008), pp. 2910-2912, ISSN 0003-6951.

Zenkevich, A., Lebedinskii, Yu., Antonov, D., Gorshkov, O. \& Filatov, D. (2011). Structure and electron transport in the ultrathin nanocomposite $\mathrm{SiO}_{2} / \mathrm{Si}$ films with the metal nanoclusters. In: Advances in Diverse Industrial Applications of Nanocompositess, B. Reddy, pp. 317-340, InTech, ISBN 978-953-307-202-9, Vienna. 


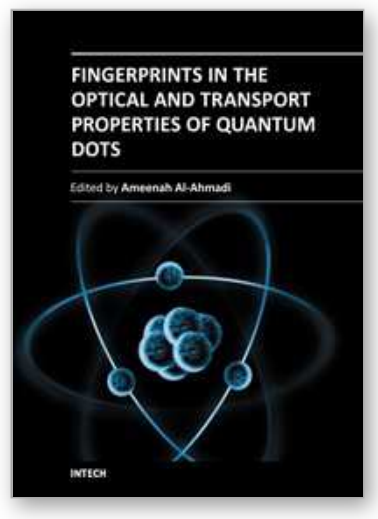

\section{Fingerprints in the Optical and Transport Properties of Quantum Dots}

Edited by Dr. Ameenah Al-Ahmadi

ISBN 978-953-51-0648-7

Hard cover, 468 pages

Publisher InTech

Published online 13, June, 2012

Published in print edition June, 2012

The book "Fingerprints in the optical and transport properties of quantum dots" provides novel and efficient methods for the calculation and investigating of the optical and transport properties of quantum dot systems. This book is divided into two sections. In section 1 includes ten chapters where novel optical properties are discussed. In section 2 involve eight chapters that investigate and model the most important effects of transport and electronics properties of quantum dot systems This is a collaborative book sharing and providing fundamental research such as the one conducted in Physics, Chemistry, Material Science, with a base text that could serve as a reference in research by presenting up-to-date research work on the field of quantum dot systems.

\section{How to reference}

In order to correctly reference this scholarly work, feel free to copy and paste the following:

Dmitry Filatov, Vladimir Shengurov, Niyaz Nurgazizov, Pavel Borodin and Anastas Bukharaev (2012). Tunneling Atomic Force Microscopy of Self-Assembled In(Ga)As/GaAs Quantum Dots and Rings and of GeSi/Si(001) Nanoislands, Fingerprints in the Optical and Transport Properties of Quantum Dots, Dr. Ameenah Al-Ahmadi (Ed.), ISBN: 978-953-51-0648-7, InTech, Available from: http://www.intechopen.com/books/fingerprints-in-the-optical-and-transport-properties-of-quantumdots/tunneling-atomic-force-microscopy-of-self-assembled-in-ga-as-gaas-quantum-dots-and-rings-and-of-

\section{INTECH}

open science | open minds

\author{
InTech Europe \\ University Campus STeP Ri \\ Slavka Krautzeka 83/A \\ 51000 Rijeka, Croatia \\ Phone: +385 (51) 770447 \\ Fax: +385 (51) 686166 \\ www.intechopen.com
}

\author{
InTech China \\ Unit 405, Office Block, Hotel Equatorial Shanghai \\ No.65, Yan An Road (West), Shanghai, 200040, China \\ 中国上海市延安西路65号上海国际贵都大饭店办公楼 405 单元 \\ Phone: +86-21-62489820 \\ Fax: $+86-21-62489821$
}


(C) 2012 The Author(s). Licensee IntechOpen. This is an open access article distributed under the terms of the Creative Commons Attribution 3.0 License, which permits unrestricted use, distribution, and reproduction in any medium, provided the original work is properly cited. 